\title{
Some Results on Fixed and Best Proximity Points of Precyclic Self-Mappings
}

\author{
M. De la Sen \\ Institute of Research and Development of Processes, University of Basque Country, Campus of Leioa (Bizkaia)-Aptdo. Postal 644-Bilbao, \\ 48080 Bilbao, Spain
}

Correspondence should be addressed to M. De la Sen; manuel.delasen@ehu.es

Received 11 March 2013; Accepted 2 July 2013

Academic Editor: D. R. Sahu

Copyright (C) 2013 M. De la Sen. This is an open access article distributed under the Creative Commons Attribution License, which permits unrestricted use, distribution, and reproduction in any medium, provided the original work is properly cited.

This paper is devoted to investigating the limit properties of distances and the existence and uniqueness of fixed points, best proximity points and existence, and uniqueness of limit cycles, to which the iterated sequences converge, of single-valued, and socalled, contractive precyclic self-mappings which are proposed in this paper. Such self-mappings are defined on the union of a finite set of subsets of uniformly convex Banach spaces under generalized contractive conditions. Each point of a subset is mapped either in some point of the same subset or in a point of the adjacent subset. In the general case, the contractive condition of contractive precyclic self-mappings is admitted to be point dependent and it is only formulated on a complete disposal, rather than on each individual subset, while it involves a condition on the number of iterations allowed within each individual subset before switching to its adjacent one. It is also allowed that the distances in-between adjacent subsets can be mutually distinct including the case of potential pairwise intersection for only some of the pairs of adjacent subsets.

\section{Introduction}

A relevant attention has been recently devoted to the research of existence and uniqueness of fixed points of self-mappings as well as to the investigation of associate relevant properties like, for instance, stability of the iterations [1-3]. The extension of such topics to the existence of either fixed points of multivalued self-mappings [1, 4-19], in generalized metric spaces $[20,21]$, or to the existence of common fixed points of several multivalued mappings or operators is receiving an important attention, for example, $[7,8,15-$ $19,22]$ and references therein. Relevant properties on the existence and uniqueness of fixed points and best proximity points for multivalued cyclic self-mappings have been studied under general contractive-type conditions based on the Hausdorff metric between subsets of a metric space. See, for instance, [4, 7-9], including as a relevant particular case the contractive condition on contractive single-valued selfmappings, [1,4-10], as well as concerns related to their extension to cyclic self-mappings. See, for instance, $[7,8$,
11] and references there in. The various related performed researches include the cases of strict contractive cyclic selfmappings and Meir-Keeler type cyclic contractions [23, 24]. Also, some of the existing background fixed point results on contractive single and multivalued self-mappings, [1, $4,5,9,10,25-28]$ and references therein, under some types of contractive conditions, have been revisited and extended in [4]. There is also a wide sample of fixed point type results available on fixed points and asymptotic properties of the iterations for self-mappings satisfying a number of contractive-type conditions while being endowed with partial order conditions. See [18, 19] and references therein.

The main objective of this paper is the investigation of the properties of the distances as well as the existence and uniqueness of fixed points and best proximity points related to contractive so-called single-valued contractive $p(\geq 2)$-precyclic self-mappings $T: \bigcup_{i \in \bar{p}} A_{i} \rightarrow \bigcup_{i \in \bar{p}} A_{i}$. Such a concept extends that of contractive $p(\geq 2)$-cyclic selfmappings. 
The concept of precyclic self-mapping generalizes that of cyclic self-mappings in the sense that a finite set of consecutive iterations are optionally allowed within a particular subset of the cyclic disposal of interest before a switching of the image of the self-mapping to the adjacent subset of its pre-image in the iterated sequence. It can also eventually happen that some sequence enters a certain subset and the solution remains permanent within such a subset. The precyclic self-mappings are contractive if they are subject to contractive conditions of similar types to those arising in contractive cyclic self-mappings.

Precyclic contractive self-mappings allow the generation of iterated sequences under constraints of the form $T^{j}\left(A_{i}\right) \subseteq$ $A_{i} \cup A_{i+1}$ for $j=j(i, x)$ being less than a prescribed positive integer number $\bar{j}=\bar{j}(i, x)$; for all $x \in \bigcup_{i \in \bar{p}} A_{i}$, for all $i \in \bar{p}$ which can be set and point dependent, while $T^{\bar{j}}\left(A_{i}\right) \subseteq A_{i+1}$; for all $i \in \bar{p}$. The ordering of the subsets for switching between pairs of adjacent subsets to perform the $p$-precyclic selfmapping is, so-called, in the sequel a $p$-precyclic disposal.

Let $\mathbf{R}_{0+}=\mathbf{R}_{+} \cup\{0\}$ be the set of nonnegative real numbers and $\mathbf{Z}_{0+}=\mathbf{Z}_{+} \cup\{0\}$ the set of nonnegative integer numbers. Consider a metric space $(X, d)$ endowed with a metric $d: X \times X \rightarrow \mathbf{R}_{0+}$ and a finite set of nonempty subsets $A_{i} ; i \in \bar{p}=\{1,2, \ldots p\}$ of $X$ and a so-called $p(\geq 2)$ precyclic self-mapping $T: \bigcup_{i \in \bar{p}} A_{i} \rightarrow \bigcup_{i \in \bar{p}} A_{i}$ such that $T\left(A_{i}\right) \subseteq A_{i} \cup A_{i+1}$; for all $i \in \bar{p}$, where $A_{i}=A_{j}$ for $i, j \in \bar{p}$ under the congruence relation $j \equiv i(\bmod p)$, that is $A_{p n+i}=A_{i}$; for all $n \in \mathbf{Z}_{0+}$, for all $i \in \bar{p}$. Note that the previous concept of precyclic self-mapping generalizes that of a $p$-cyclic self-mapping which satisfies the stronger constraint $T\left(A_{i}\right) \subseteq A_{i+1}$; for all $i \in \bar{p}$. Let $D_{i, j}=d\left(A_{i}, A_{j}\right)$ be the distance in-between any two subsets $A_{i}, A_{j} \subset X$; for all $i, j \in \bar{p}$. Note that, compared to a cyclic self-mapping, an iterated sequence from a precyclic self-mapping might contain iterated subsequences of finite or infinite cardinals in a single subset $A_{i} \subset X(i \in \bar{p})$ even if $\bigcap_{i \in \bar{p}} A_{i} \neq \varnothing$. Also, certain iterated sequences generated from $p$-precyclic selfmappings can converge to a fixed point, rather than oscillate in-between sets of distinct best proximity points, even if $\bigcap_{i \in \bar{p}} A_{i}=\varnothing$, provided that the iterated points all stay in the same subset $A_{i} \subset X$, for some $i \in \bar{p}$, after a finite number of iterations.

For each given $x \in A_{i}$, define the following nondecreasing strictly ordered (in general, point dependent) switching sequence of nonnegative integers:

$$
\begin{array}{r}
S_{i}^{*}(x)=\left\{j_{i-1}^{*}(x)=0, j_{i}^{*}(x), j_{i+1}^{*}(x), \ldots, j_{p}^{*}(x),\right. \\
\left.j_{1+p}^{*}(x), \ldots, j_{i+j+\ell p}^{*}(x), \ldots\right\} ; \quad \forall i \in \bar{p}
\end{array}
$$

containing the numbers of consecutive iterations within each individual subset $A_{i} \subseteq X$;for all $i \in \bar{p}$, before switching to the successive adjacent subsets $A_{i+1}, A_{i+2}$, $\ldots, A_{i-1}, A_{i}, A_{i+1}, A_{i+2}, \ldots$, and so forth for $j \in \bar{p}$ of the iterated sequence

$$
\begin{aligned}
& P_{i}(x) \\
& =\left\{\left\{T^{j_{i-1}^{*}(x)} x(=x),\right.\right. \\
& \left.\quad T^{j_{i-1}^{*}+1(x)} x(=T x), \ldots, T^{j_{i}^{*}(x)-1} x\right\}\left(\subseteq A_{i}\right), \ldots, \\
& \left.\quad\left\{T^{j_{i+j+\ell p}^{*}(x)} x, \ldots, T^{j_{i+j+\ell p+1}^{*}(x)-1} x\right\}\left(\subseteq A_{i+j+1}\right), \ldots\right\} .
\end{aligned}
$$

For all $i \in \bar{p}$ such that

$$
j_{i+j+\ell p}^{*}(x)=\left\{\begin{array}{l}
\min \left(k(\geq j+\ell p) \in \mathbf{Z}_{0+}: T^{k} x \in A_{i+j+1} \backslash A_{i+j}\right) ; \quad \forall i \in \bar{p}, \quad \forall \ell \in \mathbf{Z}_{0+}, \quad \text { if } 0 \leq j \leq p-i-1 \\
\min \left(k(\geq j+\ell p) \in \mathbf{Z}_{0+}: T^{k} x \in A_{i+j+1-p} \backslash A_{i+j-p}\right) ; \quad \forall i \in \bar{p}, \quad \forall \ell \in \mathbf{Z}_{0+}, \quad \text { if } p \geq j>p-i-1
\end{array}\right.
$$

for any given $x \in A_{i}$ and, either $j_{k+1}^{*}(x)-j_{k}^{*}(x)<\infty$; for all $k \in \mathbf{Z}_{0+}$ with $\operatorname{card} S_{i}^{*}(x)=\chi_{0}$ (i.e., infinite cardinal of a numerable set) or there is $j_{k^{*}(x)}^{*}(x)<\infty$ for some existing finite $k^{*}=k^{*}(x) \in \mathbf{Z}_{0+}$ and then $S_{i}^{*}(x)=\left\{j_{i-1}^{*}(x)=\right.$ $\left.0, j_{i}^{*}(x), j_{i+1}^{*}(x), \ldots, j_{k^{*}(x)}^{*}(x)<\infty\right\}$ is a finite set, that is, $\operatorname{card} S_{i}^{*}(x)=k^{*}(x)-i(x)+2<\chi_{0}$ with $T^{j_{k^{*}(x)}^{*}+k} x$ being in the same subset $A_{i} \subset X$ as $T^{j_{k^{*}(x)}^{*}} x$; for all $k\left(\leq \operatorname{card} S_{i}^{*}(x)\right) \in \mathbf{Z}_{0+}$; for all $i \in \bar{p}$. If card $S_{i}^{*}(x)=1$ for any $x \in \bigcup_{i \in \bar{p}} A_{i}$, for all $i \in \bar{p}$, then only one iteration stays at each subset before switching to the adjacent one so that the $p$-precyclic self-mapping is a standard $p$-cyclic one. Note, for instance, that if $j_{i}^{*}(x)=1$ in (2) and $x \in A_{i}$, then $T x \in A_{i+1}$. If this occurs for each $x \in \bigcup_{i \in \bar{p}} A_{i}$, then $T$ on $\bigcup_{i \in \bar{p}} A_{i}$ is a usual $p$-precyclic selfmapping.

We will establish the formulation in metric spaces $(X, d)$. It might be pointed out that it is usual to state formulations related to differential or dynamic systems and their stability, including those being formulated in a fractional calculus framework, in normed or Banach spaces since their dynamics evolve through time described by their state vectors [14, 2939]. A possibility to focus on the study of their equilibrium points in a formal and structured fashion as well as their limit solutions, provided that they exist, (for instance, the presence of possible limit cycles) is through fixed point theory since the equilibrium points are fixed points of certain mappings and the limit cycles are repeated portions of limit state space trajectories. See, for instance, [33] and references therein. In the subsequently formulated and proved results, where the convexity of sets of $X$ is required, it will be assumed that $(X,\|\|)$ is a normed space with associated metric space $(X, d)$ for a norm-induced metric $d: X \times X \rightarrow \mathbf{R}_{0+}$, [29]. If $(X,\|\|)$ is a Banch space, then $(X, d)$ is a complete metric space. The converses are not true without invoking additional assumptions. For instance, if $(X, d)$ is a metric space (resp., a complete metric space) endowed with a homogeneous and 
translation -invariant metric $d: X \times X \rightarrow \mathbf{R}_{0+}$, then the metric-induced norm \|\| exists so that $(X,\|\|)$ is a normed (resp., Banach) space endowed with such a metric-induced norm.

Example 1. For some given $\varepsilon \in \mathbf{R}_{0+}$, define the real intervals

$$
\begin{aligned}
& A_{1}=\mathbf{R}_{\varepsilon+}=\{z \in \mathbf{R}: z \geq \varepsilon\} ; \\
& A_{2}=\mathbf{R}_{\varepsilon-}=\{z \in \mathbf{R}: z \leq-\varepsilon\}
\end{aligned}
$$

and consider the scalar sequence

$$
x_{n+1}= \begin{cases}\widehat{x}_{n+1}, & \text { if }\left|\widehat{x}_{n+1}\right| \geq \varepsilon \\ -\varepsilon \operatorname{sgn} x_{n}, & \text { otherwise }\end{cases}
$$

where

$$
\begin{array}{r}
\widehat{x}_{n+1}=(-1)^{n+1} a_{n} x_{n} ; \quad \forall n \in \mathbf{Z}_{0+} \text { with initial condition } \\
\text { satisfying }\left|x_{0}\right| \geq \varepsilon
\end{array}
$$

with $\left\{a_{n}\right\} \subset \mathbf{R}_{0+}$. Note that, if $\varepsilon=0$, then $A_{1} \cap A_{2}=\{0\}$ and $\{0\}$ can be a candidate for fixed point depending on certain simple contractive or, at least, nonexpansive conditions on the sequence $\left\{a_{n}\right\}$. If $\varepsilon \neq 0$, then the convergence of the sequences $\left\{x_{2 n}\right\}$ and $\left\{x_{2 n+1}\right\}$ (but not that of $\left\{x_{n}\right\}$ which is not convergent) can be possible only to best proximity points $\pm \varepsilon$. The self-mapping $T$ on $A_{1} \cup A_{2}$ defining the solution sequence is a 2 -cyclic one since the solution points are alternately in $A_{1}$ and $A_{2}$. However, the modification

$$
x_{n+1+i}= \begin{cases}\widehat{x}_{n+1+i}, & \text { if }\left|\widehat{x}_{n+1}\right| \geq \varepsilon, \\ -\varepsilon v_{n+i} \operatorname{sgn} x_{n}, & \text { otherwise, }\end{cases}
$$

where

$$
\begin{gathered}
\widehat{x}_{n+1+i}=(-1)^{n+1} v_{n+i} a_{n} x_{n} \\
v_{n+i}= \begin{cases}(-1)^{n+1}, & \text { if } 0 \leq i \leq j^{*}=2, \\
1, & \text { if } i=j^{*} .\end{cases}
\end{gathered}
$$

For all $n \in \mathbf{Z}_{0+}$ with initial value $\left|x_{0}\right| \geq \varepsilon$ is a 2 -precyclic (but no a 2-cyclic) self-mapping which generates two consecutive iterations in both $A_{1}$ and $A_{2}$ before switching to the other subset. Several extended variants are possible; that is, $j^{*}=$ $j_{0}^{*}=j_{1}^{*}=2$ is constant in this case. For instance, $j^{*}$ can be dependent on the solution point $x(n)$ or on the initial condition. If $j^{*}\left(x\left(n_{0}\right)\right)$ is infinity, then the trajectory solution remains in either $A_{1}$ (resp., in $A_{2}$ ) for $n \geq n_{0}$ if $x\left(n_{0}\right) \in$ $A_{1}$ (resp., $x\left(n_{0}\right) \in A_{2}$ ). In this case, depending on conditions on the parameterization sequence $\left\{a_{n}\right\}$, the convergence of the solution in one of the subsets could be possible, even if $\varepsilon \neq 0$, when $j^{*}$ is infinity in at least one of the sets $A_{1}$ and $A_{2}$ for some subset of values of the solution so that the solution enters such a set and remains in it for all later iterations. If $j^{*}=1$ for any point of the solution sequence at any iteration, then the solution trajectory switches in-between the subsets $A_{1}$ and $A_{2}$ so that the 2-precyclic self-mapping is also a 2cyclic one.

\section{Convergence of Iterated Sequences to Fixed Points}

The following assumptions are made.

(1) There are $p$ bounded real functions $K_{i}: A_{i} \rightarrow K_{i} \epsilon$ $\left(0, \bar{K}_{i}\right]$; for all $i \in \bar{p}$ fulfilling $K_{i}(x)=K_{j}(x)$ in $\bigcup_{i \in \bar{p}} A_{i}$ under the congruence relation $j \equiv i(\bmod p)$ for some $i=i(j) \in \bar{p}$ and any given $j \in \mathbf{Z}_{+}$such that

$$
d\left(T x, T^{2} x\right) \leq K_{i}(x) d(x, T x)+\left(1-K_{i}(x)\right) v_{i}(x) D_{i}
$$

for $x \in A_{i}$ where $v_{i}: A_{i} \rightarrow\{0,1\}$ are binary functions; for all $i \in \bar{p}$ such that $v_{i}(x)=0$ if $T x \in A_{i}$ and $v_{i}(x)=1$ if $T x \in A_{i+1} \backslash A_{i}$; for all $x \in A_{i}$, for all $i \in \bar{p}$. The notation to be used does not distinguish explicitly the cases when the contractive-like functions are real constants or pointdependent functions, but this can be easily deduced from context. $i \in \bar{p}$

(2) If $D_{i}>0$, that is, if $A_{i} \cap A_{i+1}=\varnothing$, then $\bar{K}_{i}<1$; for all

(3) If $\operatorname{card} S^{*}(x)<\chi_{0}$, then $K_{k^{*}(x)} \leq 1$, where $k^{*}(x) \equiv i+$ $j(\bmod p)$; for all $x \in A_{i}$, for all $i \in \bar{p}$, provided that $A_{i+j}$ is closed

(4)

$$
\limsup _{k \rightarrow \infty}\left(\sum_{j=0}^{k}\left[K_{i+j}^{j_{i+j}^{*}(x)}\right]\right) \leq 1 ; \quad x \in \bigcup_{i \in \bar{p}} A_{i}, \forall i, j \in \bar{p} .
$$

If $(x, y \neq T x) \in A_{i} \times A_{i+1}$ for some $i \in \bar{p}$, then the constraint (9) is replaced with its following standard counterpart stated for $p$-cyclic self-mappings:

$$
d(T x, T y) \leq K_{i}(x) d(x, y)+\left(1-K_{i}(x)\right) D_{i} .
$$

Note that the previous condition holds if $T x \in A_{i+1}$ and $T y \in A_{i+2}$ but also if $T x, T y \in A_{i+1}$ in its particular version $d(T x, T y) \leq K_{i}(x) d(x, y)$, since $D_{i} \geq 0$, and that it contains (9) for iterated sequences generated from $T: \bigcup_{i \in \bar{p}} A_{i} \rightarrow$ $\bigcup_{i \in \bar{p}} A_{i}$ as a particular case. Note also the following.

(a) A particular pair $(x, T x)$ can satisfy simultaneously several constraints (9). For instance, assume that $x, T x \in A_{i} \cap A_{i+1}(\neq \varnothing)$ for some $i \in \bar{p}$. Then

$$
d\left(T x, T^{2} x\right) \leq \min \left(K_{i}(x), K_{i+1}(x)\right) d(x, T x) .
$$

(b) If $\operatorname{card} S^{*}(x)<\chi_{0}$, then there is some set $A_{j}(j \in$ $\bar{p})$ such that $T^{k} x \subseteq A_{j}$; for all $k \geq k_{0}$ and some finite $k_{0}=k_{0}(x) \in \mathbf{Z}_{0+}$ for each given $x \in \bigcup_{i \in \bar{p}} A_{i}$. Then, $T: \bigcup_{i \in \bar{p}} A_{i} \rightarrow \bigcup_{i \in \bar{p}} A_{i}$ is nonexpansive and $\left\{T^{k} x\right\}_{k \in Z_{0+}}$ is bounded. Note that Assumption 4 is guaranteed directly by Assumption 3 if $\operatorname{card} S^{*}(x)<\chi_{0}$. If $\operatorname{card} S^{*}(x)=\chi_{0}$, then Assumption 3 is not invoked; however, Assumption 4 guarantees that $\left\{T^{k} x\right\}_{k \in \mathbf{Z}_{0+}}$ is bounded with $T^{k} x \in$ $\bigcup_{i \in \bar{p}} A_{i}$

(c) Assumption 4 implies that the $p$-precyclic selfmapping $T: \bigcup_{i \in \bar{p}} A_{i} \rightarrow \bigcup_{i \in \bar{p}} A_{i}$ is asymptotically nonexpansive. 
(d) Any $p$-precyclic self-mapping $T: \bigcup_{i \in \bar{p}} A_{i} \rightarrow$ $\bigcup_{i \in \bar{p}} A_{i}$ is also a $p$-cyclic self-mapping.

In the following, $\operatorname{fix}(G)$ denotes the set of fixed points of the self-mapping $G: X \rightarrow X$. The following results hold.

Theorem 2. Let $T: \bigcup_{i \in \bar{p}} A_{i} \rightarrow \bigcup_{i \in \bar{p}} A_{i}$ be a p-precyclic selfmapping. Assume also that the constraint (9) is extended for any $x \in A_{i}$ and $y \in A_{j}$; for all $i, j \in \bar{p}$ as follows:

$$
\begin{aligned}
d(T x, T y) \leq & \max \left(K_{i}(x), K_{j}(y)\right) d(x, y) \\
& +\left(1-\max \left(K_{i}(x), K_{j}(y)\right)\right) \nu_{i j}(x) D_{i j},
\end{aligned}
$$

where $D_{i j}=d\left(A_{i}, A_{j}\right)$ and $v_{i j}(x)=1$ if $T x \in A_{i+1} \backslash A_{i}$ and Ty $\in A_{j+1} \backslash A_{j}$ and $v_{i j}(x)=0$, otherwise; for all $x \in A_{i}$, for all $y \in A_{j}$.

Then, the following properties hold.

(i) $T: \bigcup_{i \in \bar{p}} A_{i} \rightarrow \bigcup_{i \in \bar{p}} A_{i}$ is a $p$-cyclic self-mapping if and only if $T\left(A_{i}\right) \subseteq A_{i+1}$; for all $i \in \bar{p}$.

(ii) If $D_{i}>0$; for all $i \in \bar{p}$ and card $S^{*}(x)=\chi_{0}$; for all $x \in \bigcup_{i \in \bar{p}} A_{i}$, then $T: \bigcup_{i \in \bar{p}} A_{i} \rightarrow \bigcup_{i \in \bar{p}} A_{i}$ has no fixed point in $\bigcup_{i \in \bar{p}} A_{i}$.

(iii) If card $S^{*}(x)<\chi_{0}$ for some $x \in \bigcup_{i \in \bar{p}} A_{i}$, then $T: \bigcup_{i \in \bar{p}} A_{i} \rightarrow \bigcup_{i \in \bar{p}} A_{i}$ has a fixed point in a subset $A_{j} \subset X$, for some $j \in \bar{p}$, to which the iterated sequence $\left\{x, T x, \ldots, T^{k} x, \ldots\right\}$ converges if $(X, d)$ is complete, $K_{j}<1$ and $A_{j}$ is closed. If $x \in A_{i}$ for some $i \in \bar{p}$, then the iterated sequence $\left\{x, T x, \ldots, T^{k} x, \ldots\right\}$ converges to a fixed point $x_{i+j}^{*} \in A_{i+j}$ of $T: \bigcup_{i \in \bar{p}} A_{i} \rightarrow \bigcup_{i \in \bar{p}} A_{i}$ for some $j \in \overline{p-i}$ with $K_{i+j}<1$ such that $k^{*}(x) \equiv$ $i+j(\bmod p)$, provided that $A_{i+j}$ is closed, or to a fixed point $x_{j}^{*} \in A_{j}$ for some $j \in \overline{i-1}$ with $K_{j}<1$ and $k^{*}(x) \equiv i+j(\bmod p)$ provided that $A_{j}$ is closed. If, furthermore, $x_{i+j}^{*} \in A_{i+j}$ for $j \in \overline{p-i}$, respectively, $x_{j}^{*} \in A_{j}$ for $j \in \bar{i}$ are convex then the corresponding fixed point is unique.

Proof. $T\left(A_{i}\right) \cap A_{i} \neq \varnothing$ for some $i \in \bar{p}$; then $\exists x \in A_{i}$ such that $T x \notin A_{i+1}$. Then, $T\left(A_{i}\right) \subseteq A_{i} \cup A_{i+1}$ is not a cyclic selfmapping. Hence, Property (i) follows.

Note that $D_{i}>0$; for all $i \in \bar{p} \Leftrightarrow A_{i} \cap A_{i+1}=\varnothing$; for all $i \in \bar{p}$. If $\operatorname{card} S^{*}(x)=\chi_{0}$, then $S^{*}(x)$ is an infinite sequence of switches in-between adjacent subsets of $X$ which are never intersecting. Thus, $T: \bigcup_{i \in \bar{p}} A_{i} \rightarrow \bigcup_{i \in \bar{p}} A_{i}$ has no fixed point in $\bigcup_{i \in \bar{p}} A_{i}$. Hence, Property (ii) follows.

To prove Property (iii), first note that

$$
A_{i+j+\ell p}=A_{i+j}=A_{k} ; \quad \forall \ell \in \mathbf{Z}_{0+} ; \forall i, j \in \bar{p}
$$

such that

$$
\begin{aligned}
i+j+\ell p & \equiv i+j(\bmod p) \\
& \equiv k\left(=i+j-p ; k \in \mathbf{Z}_{+}\right)(\bmod p) .
\end{aligned}
$$

Note also that, if $\operatorname{card} S^{*}(x)<\chi_{0}$ for some $x \in \bigcup_{i \in \bar{p}} A_{i}$, then the iterated sequence $S(x)=\left\{x, T x, \ldots, T^{k} x, \ldots\right\}$ built from such an initial point $x \in \bigcup_{i \in \bar{p}} A_{i}$ through $T$ : $\bigcup_{i \in \bar{p}} A_{i} \rightarrow \bigcup_{i \in \bar{p}} A_{i}$ remains in $A_{j} \subset X$, for such a $j \in \bar{p}$, for all $k \geq j_{k^{*}(x)}^{*}$ and some finite integer $j_{k^{*}(x)}^{*}$. Then, the asymptotically nonexpansive self-mapping $T: \bigcup_{i \in \bar{p}} A_{i} \rightarrow$ $\bigcup_{i \in \bar{p}} A_{i}$ is asymptotically contractive from Assumption 4 and also contractive after a finite number of iterations since $K_{j}<1$. Thus, $S(x)$ is bounded and has a Cauchy convergent subsequence since $(X, d)$ is complete. Since the subset $A_{j} \subset X$ is nonempty and closed for such $j \in \bar{p}$, there is a fixed point of $T: \bigcup_{i \in \bar{p}} A_{i} \rightarrow \bigcup_{i \in \bar{p}} A_{i}$ from Banach contraction principle and such a fixed point is unique if the subset is, furthermore, convex.

Now, the following result is proven for a class of contractive $p$-precyclic self-mapping $T: \bigcup_{i \in \bar{p}} A_{i} \rightarrow \bigcup_{i \in \bar{p}} A_{i}$.

Theorem 3. Assume that $(X,\|\|)$ is a normed space with associated metric space $(X, d)$ for a norm-induced metric $d$ : $X \times X \rightarrow \mathbf{R}_{0+}$. Let $T: \bigcup_{i \in \bar{p}} A_{i} \rightarrow \bigcup_{i \in \bar{p}} A_{i}$ be a $p$ precyclic self-mapping and $D_{i}=0$; for all $i \in \bar{p}$. If $A_{i} \subset X$ are nonempty, bounded, closed, and convex; for all $i \in \bar{p}$ and $\bar{K}=\prod_{i \in \bar{p}}\left[\bar{K}_{i}^{j_{i}^{*}}\right]<1$, where $\bar{j}_{i}^{*}=\sup _{x \in A_{i}} j_{i}^{*}(x)$. Then $T$ : $\bigcup_{i \in \bar{p}} A_{i} \rightarrow \bigcup_{i \in \bar{p}} A_{i}$ has a unique fixed point $x^{*} \in \bigcap_{i \in \bar{p}} A_{i}$. If $A_{i} \subset X$ is not closed for some $i \in \bar{p}$ while $(X,\|\|)$ is a Banach space, and then $(X, d)$ is a complete metric space, then $T: \bigcup_{i \in \bar{p}} A_{i} \rightarrow \bigcup_{i \in \bar{p}} A_{i}$ has a unique fixed point in $\bigcap_{i \in \bar{p}} c l A_{i}$.

Proof. It follows that

$$
\begin{array}{r}
\lim _{n \rightarrow \infty} d\left(T^{p n \bar{j}^{*}} x, T^{p n \bar{j}^{*}-1} x\right) \leq \lim _{n \rightarrow \infty}\left(\bar{K}^{n}\right) d(T x, x)=0 ; \\
\forall x \in \bigcup_{i \in \bar{p}} A_{i}
\end{array}
$$

since $d(x, T x)<\infty$ for any finite $x \in \bigcup_{i \in \bar{p}} A_{i}$, since $\bigcup_{i \in \bar{p}} A_{i}$ is bounded, where

$$
\begin{aligned}
& \bar{j}^{*}=\sum_{i=1}^{p} \bar{j}_{i}^{*} \\
&=\max _{i \in \bar{p}} \sup _{x \in A_{i}}\left(j_{i}^{*}(x)+j_{i+1}^{*}\left(T^{j_{i}^{*}+1} x\right)+\cdots+j_{p}^{*}\left(T^{\sum_{j=i}^{p-1} j_{j}^{*}+1} x\right)\right. \\
&+j_{1}^{*}\left(T^{\sum_{j=i}^{p} j_{j}^{*}+1} x\right) \\
&\left.+\cdots+j_{i-1}^{*}\left(T^{\sum_{j=i}^{p} j_{j}^{*}+\sum_{j=p-i+1}^{p-1} j_{j}^{*}+1} x\right)\right) ;
\end{aligned}
$$

that is, the distance between any two consecutive elements of any such a sequence converges asymptotically to zero. Furthermore, since the subsets $A_{i} \subset X$ are nonempty, closed, all of them intersect and the composite self-mapping $T^{p \bar{j}^{*}}$ : $\bigcup_{i \in \bar{p}} A_{i} \rightarrow \bigcup_{i \in \bar{p}} A_{i}$ is uniformly Lipschitz -continuous in $\bigcup_{i \in \bar{p}} A_{i}$, since it is contractive with constant $\bar{K}<1$, the 
sequence $\left\{T^{p n \vec{j}^{*}} x\right\}$ converges to $x_{i}^{*}=x_{i}^{*}(x) \in A_{i}$ for some $i \in \bar{p}$, which is a unique fixed point of the composite $T^{p^{-*}}$ : $\bigcup_{i \in \bar{p}} A_{i} \rightarrow \bigcup_{i \in \bar{p}} A_{i}$ in $A_{i}$; for all $i \in \bar{p}$. To prove uniqueness, proceed by contradiction. Assume that there are two fixed points $x_{i}^{*}=T^{p^{*}} x_{i}^{*}, y_{i}^{*}\left(\neq x_{i}^{*}\right)=T^{p^{*}} y_{i}^{*}$ in $A_{i}$ of $T^{p^{*}{ }^{*}}$ : $\bigcup_{i \in \bar{p}} A_{i} \rightarrow \bigcup_{i \in \bar{p}} A_{i}$.

Then,

$$
\begin{aligned}
d\left(x_{i}^{*}, y_{i}^{*}\right)= & d\left(T^{p n \bar{j}} x_{i}^{*}, T^{p n \bar{j}^{*}} y_{i}^{*}\right) \\
\leq & d\left(T^{p n \bar{j}^{*}+1} x_{i}^{*}, T^{p n \bar{j}^{*}} x_{i}^{*}\right) \\
& +d\left(T^{p n \bar{j}^{*}+1} x_{i}^{*}, T^{p n \bar{j}^{*}+1} y_{i}^{*}\right) \\
& +d\left(T^{p n \bar{j}^{*}+1} y_{i}^{*}, T^{p n \bar{j}^{*}} y_{i}^{*}\right) \\
\leq & \bar{K}^{n}\left(d\left(T x_{i}^{*}, x_{i}^{*}\right)+d\left(T x_{i}^{*}, T y_{i}^{*}\right)+d\left(T y_{i}^{*}, y_{i}^{*}\right)\right) \\
= & \bar{K}^{n} d\left(x_{i}^{*}, y_{i}^{*}\right)<d\left(x_{i}^{*}, y_{i}^{*}\right) ; \quad \forall n \in \mathbf{Z}_{+}
\end{aligned}
$$

which leads to the contradiction that $x_{i}^{*}=y_{i}^{*}$. Thus, there is a unique fixed point of $T^{\overline{p j}^{*}}: \bigcup_{i \in \bar{p}} A_{i} \rightarrow \bigcup_{i \in \bar{p}} A_{i}$ in $A_{i}$. Also, one gets from (16) that

$$
\begin{aligned}
& \lim _{n \rightarrow \infty} d\left(T^{p n \bar{j}^{*}+j} x, T^{p n \bar{j}^{*}+j-1} x\right) \\
& \quad \leq \lim _{n \rightarrow \infty}\left(\bar{K}^{n}\right)\left(\prod_{\ell=1}^{j}\left[\bar{K}_{\ell}\right]\right) d(T x, x)=0 ; \quad \forall j \in \bar{p}
\end{aligned}
$$

for any $j \in \bar{p}$. As a result,

$\lim _{n \rightarrow \infty} d\left(T^{p n \bar{j}^{*}+j} x, T^{p \bar{j}^{*}+j-1} x\right)=0$ and $\left\{T^{p \bar{j}^{*}+j} x\right\} \rightarrow x_{i}^{*}=$ $T^{p \vec{j}^{*}} x_{i}^{*}$ with some unique $x_{i}^{*} \in A_{i}$ for some $i \in \bar{p}$; for all $j \in$ $\bar{p}$. Now, assume that there are two distinct fixed points $x_{i}^{*} \in$ $A_{i}$ and $x_{j}^{*}\left(\neq x_{i}^{*}\right) \in A_{j}\left(\neq A_{i}\right)$ of for some $i, j(\neq i) \in \bar{p}$. Since $A_{i} \subseteq X$ is nonempty, closed, and convex for any $i \in \bar{p}$, then $A_{i} \cap A_{j}$ is nonempty, closed, and convex; for all $i, j(\neq i) \in \bar{p}$. From the convexity of $A_{i} \cap A_{j}$, there is $z\left(\neq x_{i}^{*}, x_{j}^{*}\right) \in A_{i} \cap A_{j}$ such that $d\left(x_{i}^{*}, z\right)=\lambda d\left(x_{i}^{*}, x_{j}^{*}\right)$ with some real constant $\lambda \epsilon$ $(0,1)$ and $x_{i}^{*}, z \in A_{i}$. Then,

$$
\begin{aligned}
& d\left(x_{i}^{*}, T^{p n \bar{j}^{*}} z\right) \\
& \quad=d\left(T^{p n \bar{j}^{*}} x_{i}^{*}, T^{p n \vec{j}^{*}} z\right) \leq \bar{K}^{n} d\left(x_{i}^{*}, z\right)=\lambda \bar{K}^{n} d\left(x_{i}^{*}, x_{j}^{*}\right)
\end{aligned}
$$

so that $d\left(x_{i}^{*}, T^{p n \bar{j}^{*}} z\right) \rightarrow 0$ as $n \rightarrow \infty$ and, since $T^{p \bar{j}^{*}}$ : $\bigcup_{i \in \bar{p}} A_{i} \rightarrow \bigcup_{i \in \bar{p}} A_{i}$ is uniformly Lipschitz continuous in $\bigcup_{i \in \bar{p}} A_{i}$, the sequence $\left\{T^{p n \bar{j}^{*}} z\right\}_{n \in \mathbf{Z}_{0+}}$ converges to $x_{i}^{*} \in A_{i}$. But $z \in A_{j}$ so that we can repeat the previous reasoning with $d\left(x_{j}^{*}, z\right)=\lambda^{\prime} d\left(x_{i}^{*}, x_{j}^{*}\right), x_{j}^{*}, z \in A_{j}$ and some real constant $\lambda^{\prime} \in(0,1)$ to conclude that $d\left(x_{j}^{*}, T^{p n \vec{j}^{*}} z\right) \rightarrow 0$ as $n \rightarrow \infty$ and $\left\{T^{p \bar{j}^{-*}} z\right\}_{n \in \mathbf{Z}_{0+}}$ converges to $x_{j}^{*}\left(\neq x_{i}^{*}\right) \in A_{j}$ which is a contradiction to its convergence to $x_{i}^{*} \in A_{i}$. Then, there is a unique fixed point in the nonempty, closed, and convex set $A_{i} \cap A_{j}$. By extending the same reasoning to any pair of subsets $A_{i}$ and $A_{j}$ of $X$, one concludes that the composite self-mapping $T^{\bar{p}^{*}{ }^{*}}: \bigcup_{i \in \bar{p}} A_{i} \rightarrow \bigcup_{i \in \bar{p}} A_{i}$ has a unique fixed point $x^{*} \in\left\{x^{*}\right\}=\operatorname{Fix}\left(T^{p^{*}}\right) \subseteq \bigcap_{i \in \bar{p}} A_{i}$

It remains to be proved that the unique fixed point of the composite mapping is also the unique fixed point of $T$ : $\bigcup_{i \in \bar{p}} A_{i} \rightarrow \bigcup_{i \in \bar{p}} A_{i}$. Since the subsets $A_{i} \subseteq X$ intersect, one gets from (16) that

$$
\begin{aligned}
& \left(d\left(T^{2 p \bar{j}^{*}+1} x^{*}, T^{2 p \bar{j}^{*}} x^{*}\right) \leq K^{\prime} \bar{K} d\left(T^{p \bar{j}^{*}} x^{*}, x^{*}\right)=0\right) \\
& \Longrightarrow\left(d\left(T\left(T^{2 p \bar{j}^{*}}\right) x^{*}, x^{*}\right)=d\left(T x^{*}, x^{*}\right)=0\right) \\
& \Longrightarrow\left(T x^{*}=x^{*}\right) \Longleftrightarrow\left(x^{*} \in \operatorname{Fix}\left(T^{p^{*}}\right)\right) \\
& \Longrightarrow\left(x^{*} \in \operatorname{Fix}(T)\right)
\end{aligned}
$$

since $T^{p \vec{j}^{*}} x^{*}=x^{*}$, where $K^{\prime}=\max _{i \in \bar{p}} K_{i}$. Also,

$$
\begin{aligned}
& \left(x^{*} \in \operatorname{Fix}(T)\right) \\
& \Longrightarrow\left(d\left(T x^{*}, x^{*}\right)=d\left(x^{*}, x^{*}\right)=d\left(T^{2} x^{*}, T x^{*}\right)\right. \\
& \left.\quad=\cdots=d\left(T^{n+1} x^{*}, T^{n} x^{*}\right)=0 ; \forall n \in \mathbf{Z}\right) \\
& \Longleftrightarrow\left(x^{*} \in \operatorname{Fix}\left(T^{n}\right) ; \forall n \in \mathbf{Z}_{+}\right) \Longrightarrow\left(x^{*} \in \operatorname{Fix}\left(T^{p^{*}}\right)\right)
\end{aligned}
$$

so that $x^{*} \in \operatorname{Fix}(T)$ and it is the unique fixed point of $T$ : $\bigcup_{i \in \bar{p}} A_{i} \rightarrow \bigcup_{i \in \bar{p}} A_{i}$. If some $A_{i} i \in \bar{p}$ is not closed, then all Cauchy sequences have a limit in $X$ if $(X, d)$ is complete so that there is still a unique fixed point in $\bigcap_{i \in \bar{p}} c l A_{i}$ of $T$ :

$\bigcup_{i \in \bar{p}} A_{i} \rightarrow \bigcup_{i \in \bar{p}} A_{i}$ and $T^{\overline{p j}^{*}}: \bigcup_{i \in \bar{p}} A_{i} \rightarrow \bigcup_{i \in \bar{p}} A_{i}$.

The following result is now proven for a class of nonexpansive $p$-precyclic self-mapping $T: \bigcup_{i \in \bar{p}} A_{i} \rightarrow \bigcup_{i \in \bar{p}} A_{i}$.

Corollary 4. Assume the hypothesis of Theorem 3 with the modified weaker condition $\bar{K}=\prod_{i \in \bar{p}}\left[\bar{K}_{i}^{j_{i}^{*}}\right] \leq 1$ of nonexpansive self-mapping $T: \bigcup_{i \in \bar{p}} A_{i} \rightarrow \bigcup_{i \in \bar{p}} A_{i}$. Then, $\left\{T^{n} x\right\}_{n \in \mathbf{Z}_{0+}}$ is bounded; for all $x \in \bigcup_{i \in \bar{p}} A_{i}$ there is a subsequence $\left\{T^{p \bar{j}^{*} n_{k}} x\right\}_{n_{k} \in Z_{s} \subseteq \mathbf{Z}_{0+}} \subseteq\left\{T^{n} x\right\}_{n \in \mathbf{Z}_{0+}}$, for some strictly ordered subset $Z_{s}$ of $\mathbf{Z}_{0+}$, such that $d\left(T^{p^{-*} n_{k}} x, T^{p^{-*} n_{k+1}} x\right) \rightarrow C=$ $C(x)$ as $k \rightarrow \infty$; for all $x \in \bigcup_{i \in \bar{p}} A_{i}$ for some real $C \in \mathbf{R}_{0+}$. If, in addition, $\bar{K}_{i} \leq 1$; for all $i \in \bar{p}$, then $d\left(T^{n+1} x, T^{n} x\right) \rightarrow C=$ $C(x)$ as $n \rightarrow \infty$; for all $x \in \bigcup_{i \in \bar{p}} A_{i}$.

Proof. $x \in \bigcup_{i \in \bar{p}} A_{i}$ is always finite since $\bigcup_{i \in \bar{p}} A_{i}$ is bounded and then $\left\{T^{n} x\right\}_{n \in \mathbf{Z}_{0+}} \subseteq \bigcup_{i \in \bar{p}} A_{i}$ is a bounded sequence; for all $x \in \bigcup_{i \in \bar{p}} A_{i}$. Also, $d\left(T^{p \bar{j}^{*} n} x, T^{p \bar{j}^{*} n+1} x\right) \leq d(x, T x)$; for 
all $n \in \mathbf{Z}_{0+}$, for all $x \in \bigcup_{i \in \bar{p}} A_{i}$ since $\bar{K}=\prod_{i \in \bar{p}}\left[\bar{K}_{i}^{\bar{j}_{i}^{*}}\right] \leq 1$. Thus, $\lim \sup _{n \rightarrow \infty} d\left(x, T^{n+1} x\right)<\infty$; for all $x \in \bigcup_{i \in \bar{p}} A_{i}$ since $\left\{T^{n} x\right\}_{n \in \mathbf{Z}_{0+}}$ is bounded and $d\left(T^{n} x, T^{n+1} x\right)<\infty$; for all $n \in \mathbf{Z}_{0+}$ from the properties of distances since $x \in \bigcup_{i \in \bar{p}} A_{i}$ is finite. Thus, there is a subsequence $\left\{T^{p \bar{j}^{*} n_{k}} x\right\}_{n_{k} \in Z_{s} \subseteq Z_{0+}} \subseteq$ $\left\{T^{k} x\right\}_{k \in \mathbf{Z}_{0+}}$ for which $d\left(T^{p \bar{j}^{*} n_{k}} x, T^{p^{\bar{j}^{*}} n_{k+1}} x\right)$ converges as $k \rightarrow$ $\infty$. If, in addition, $\bar{K}_{i} \leq 1$; for all $i \in \bar{p}$, then $d\left(T^{n+1} x, T^{n} x\right) \leq$ $\left(\max _{i \in \bar{p}} K_{i}\right) d(x, T x) \leq d(x, T x)$; for all $x \in \bigcup_{i \in \bar{p}} A_{i}$, so that $d\left(T^{n+1} x, T^{n} x\right) \rightarrow C=C(x)$ as $n \rightarrow \infty$; for all $x \in$ $\bigcup_{i \in \bar{p}} A_{i}$.

The subsequent result is related to convergence to a unique fixed point in one of the subsets $A_{i} \subseteq X(i \in \bar{p})$ of the precyclic disposal even if the subsets do not intersect, provided that the self-mapping is asymptotically contractive in one of the subsets.

Corollary 5. Assume the hypothesis of Theorem 3 with the subsequent further hypothesis on the p-precyclic self-mapping $T: \bigcup_{i \in \bar{p}} A_{i} \rightarrow \bigcup_{i \in \bar{p}} A_{i}:$

(1) $\bigcap_{i \in \bar{p}} A_{i}=\varnothing ; \operatorname{card}_{i}^{*}(x)<\chi_{0}$

(2) $\exists j \in J_{0}=J_{0}(x) \subseteq \bar{p}$ (nonnecessarily unique; that $i s$, $J_{0}$ can have a cardinal greater than one) such that $\lim \sup _{\ell \rightarrow \infty} K_{j}\left(T^{i+j+\ell p+1} x\right)<1$ for some given initial point $x \in A_{i}$.

Then, the iterated sequence $P_{i}(x),(2)$, converges to a fixed point in $A_{k} \subset X$ for a unique $k=\min \left(j \in J_{i<}: j \in J_{0}\right) \in \bar{p}$, where

$$
\begin{aligned}
J_{i \prec}= & J_{i \prec}(x) \\
= & \{i(\prec i+1), i+1(\prec i+2), i+2(\prec i+3), \ldots, \\
& \quad p-1(\prec p), p(\prec, 1), 1(\prec 2), \ldots, i-1 \prec(i)\}
\end{aligned}
$$

is a strictly ordered finite set of card $J_{i<}=p$, containing all the $p$ elements of the set $\bar{p}$, with the strict order relation $\prec$ defined by its enumeration order defined by $a \prec b$ for any $a, b \in J$ if $a$ precedes $b$ in the previous enumeration definition of the set $J$.

Proof. Since card $S_{i}^{*}(x)<\chi_{0} ; \exists j \in \bar{p}$ such that for the given $x \in A_{i} j_{i+j+\ell p+1}^{*}(x) \rightarrow \infty$ as $\ell \rightarrow \infty$ if $0 \leq j \leq p-i-1$, or $j_{k+\ell p}^{*}(x) \rightarrow \infty$ as $\ell \rightarrow \infty$ if $2 p \geq j>p-i-1$ for any nonnegative integer $k>i-1$ such that $j \equiv k-i-1(\bmod p)$. This is obvious since, if such aj $\in \bar{p}$ does not exist, then the iterated sequence $P_{i}(x)$, (2), with starting point $x \in A_{i}$ for the given $i \in \bar{p}$ has infinitely many switches in-between consecutive adjacent subsets $A_{i} \subset X$; then the switching sequence $S_{i}^{*}(x)$ associated with such an iterated sequence $P_{i}(x)$ is finite so that $\operatorname{card} S_{i}^{*}(x)=\chi_{0}$.

From the extra previous hypothesis 2 , there is a nonempty set $J_{0}(x) \subseteq \bar{p}$ for which $\lim \sup _{\ell \rightarrow \infty} K_{j}\left(T^{i+j+\ell p+1} x\right)<1$ for the given $x \in A_{i}$. Note that the $p$-precyclic self-mapping $T: \bigcup_{i \in \bar{p}} A_{i} \rightarrow \bigcup_{i \in \bar{p}} A_{i}$ is asymptotically contractive, for the given $x \in A_{i}$ on $A_{k} \subset X$, where $k=\min (j \in$
$\left.J_{i \prec}(x): j \in J_{0}(x)\right) \in \bar{p}$ is unique (even if $J_{0}(x)$ is of cardinal greater than one) from the fact that $J_{i<}(x)$ in (23) is of finite cardinal, strictly ordered, then with unique minimal and maximal elements which are then a unique $i \in \bar{p}$ minimum and maximum $i-1 \in \overline{p-1} \cup\{0\}$, respectively. From the previous part of this proof, it also turns out that $T: \bigcup_{i \in \bar{p}} A_{i} \rightarrow \bigcup_{i \in \bar{p}} A_{i}$ is, furthermore, (strictly) contractive on such a subset $A_{k} \subset X$ for any infinite subsequence $P_{i}\left(T^{j_{i+j+\ell p}^{*}(x)} x\right) \subseteq P_{i}(x)$. Therefore, such a subsequence $P_{i}\left(T^{j_{i+j+\ell p}^{*}(x)} x\right)$ is itself a Cauchy sequence fulfilling $\lim _{n \rightarrow \infty} d\left(T^{n} x, T^{n+1} x\right)=0$ and then being bounded and convergent in $A_{k}$ (since $\left\{T^{n} x\right\}_{n \geq j_{i+j+\ell p}^{*}(x)}$ satisfies a Lipschitzcontinuous property) to a fixed point $x^{*} \in A_{k}$, since $A_{k}$ is nonempty, bounded (then $x$ is bounded), and closed, which is unique since $A_{k}$ is convex. Since the sequence $P_{i}(x)$ contains, by construction, all the elements of the subsequence alter a finite number of iterations, it also converges to the same unique fixed point $x^{*}$.

Remark 6. (1) Note that Corollary 5 is stated for a certain iterated sequence being built from a starting point since the contractive conditions a point-to-point condition. Point-topoint contractivity-type conditions have been also used in the literature for the characterization of fixed point properties of contractive self-mappings. See, for instance, [40, 41] and references therein. It can be generalized directly under generalization for any starting point in any of the subsets or in some subset of its union. It can be reformulated, in particular, if $\lim \sup _{\ell \rightarrow \infty} K_{j}\left(T^{i+j+\ell p+1} x\right)<1$; for all $x \in A_{i}$. In such a case, it follows the convergence of the sequence of iterates to the same unique fixed point in $A_{k}$ built from any initial point $x \in A_{i}$.

(2) Note that the uniqueness of the final set $A_{k}$ from the initial set $A_{i}$, such that $x \in A_{i}$, arises from the fact that the first subset where the iterations remain after a finite number of them is the relevant one for the final reached limit if the precyclic self-mapping stops to transfer the iterated sequence to the next adjacent subset.

(3) Note that, if the convexity assumption is only made on the subset $A_{k}$, then Corollary 5 still holds.

(4) Note also that, if the convexity assumption on the subsets is removed in Corollary 5, then the existence of the fixed point is still proven although that one is not necessarily unique.

The subsequent result is a consequence of direct proof of Corollaries 4 and 5 .

Corollary 7. Assume the hypothesis of Corollary 5 under the weaker $\lim \sup _{\ell \rightarrow \infty} K_{j}\left(T^{i+j+\ell p+1} x\right) \leq 1$ condition of asymptotic nonexpansivness of the self-mapping $T: \bigcup_{i \in \bar{p}} A_{i} \rightarrow$ $\bigcup_{i \in \bar{p}} A_{i}$ in some of the subsets $A_{k} \subseteq X$ for some given initial point $x \in A_{i}$. Then, $d\left(T^{n+1} x, T^{n} x\right) \rightarrow C=C(x)$ and $\left\{T^{n} x\right\}_{n \geq n_{0}} \subseteq A_{k}$ for some finite $n_{0} \in \mathbf{Z}_{0+}$ and a unique $k \in \bar{p}$ as defined in Corollary 5. 
Some extensions of Corollary 7 can be directly obtained from Remark 6 (3)-(4).

\section{Convergence to Best Proximity Points}

The following preliminary technical result concerning the convergence for distances in-between consecutive iterates through the self-mapping $T: \bigcup_{i \in \bar{p}} A_{i} \rightarrow \bigcup_{i \in \bar{p}} A_{i}$ is now given in the case that the subsets $A_{i} \subset X$ do not necessarily intersect.

Theorem 8. Assume that the following constraints hold.

(1) $D_{i} \neq 0$; for all $i \in \bar{p}$.

(2) $K_{i} \in(0, \infty)$ is constant within each subset $A_{i} \subset X$ and the switching sequences

$$
\begin{aligned}
S_{i}^{*} & =\left\{j_{i-1}^{*}=0, j_{i}^{*}, j_{i+1}^{*}, \ldots, j_{p}^{*}, j_{1+p}^{*}, \ldots, j_{i+j+\ell p}^{*}, \ldots\right\} \\
& =\left\{j_{i-1}^{*}=0, j_{i}^{*}, S_{i+1}^{*} \backslash j_{i}^{*}\right\} ; \quad \forall i \in \bar{p}
\end{aligned}
$$

are not point dependent on the given $x \in A_{i}$; for all $i \in \bar{p}$ so that, in addition,

$$
\bar{j}_{i}^{*}=\bar{j}_{i+n p}^{*}=\sup _{x \in A_{i}} j_{i}^{*}(x)=j_{i+n p}^{*} \quad \forall n \in \mathbf{Z}_{0+} .
$$

(3)

$$
K=\prod_{i \in \bar{p}}\left[K_{i}^{\tilde{j}_{i}^{*}}\right]<1 .
$$

(4)

$$
D_{0}:=\frac{\sum_{j=1}^{p}\left(\prod_{i=j+1}^{p}\left[K_{i}^{j_{i}^{*}}\right]\right)\left(1-K_{j}\right) D_{j}}{1-\prod_{i=1}^{p}\left[K_{i}^{j_{i}^{*}}\right]} .
$$

Then, the following properties hold for any $x \in A_{i}$ :

$$
\begin{aligned}
& \limsup _{n \rightarrow \infty} d\left(T^{n\left(\sum_{i=1}^{p} j_{i}^{*}\right)+j} x, T^{n\left(\sum_{i=1}^{p} j_{i}^{*}\right)+j-1} x\right) \leq K_{i}^{j-1} D_{0} \\
& \text { for } j=1,2, \ldots, j_{i}^{*} \\
& D_{i+k} \\
& \quad \leq \limsup _{n \rightarrow \infty} d\left(T^{n\left(\sum_{i=1}^{p} j_{i}^{*}\right)+\sum_{\ell=i}^{k} j_{\ell}^{*}+j+1} x, T^{n\left(\sum_{i=1}^{p} j_{i}^{*}\right)+j_{i}^{*}+j} x\right) \\
& \quad \leq\left(\prod_{\ell=i}^{k}\left[K_{\ell}\right]\right) D_{0}+\sum_{j=i}^{k}\left(\prod_{\ell=j+1}^{k}\left[K_{\ell}^{j_{\ell}^{*}}\right]\right)\left(1-K_{j}\right) D_{j} ;
\end{aligned}
$$

If, in addition, Assumption 5

$$
\begin{gathered}
D_{1}=K_{1}^{j_{1}^{*}-1} D_{0} ; \\
D_{k}=K_{k}^{j_{k}^{*}-1}\left(\prod_{i=1}^{k-1}\left[K_{i}^{j_{i}^{*}}\right]\right) D_{0} \geq \prod_{i=1}^{k-1}\left[K_{i}^{j_{i}^{*}}\right] D_{k} \quad \text { for } k \geq 2
\end{gathered}
$$

Holds; then,

$$
\begin{array}{r}
\limsup _{n \rightarrow \infty} d\left(T^{n\left(\sum_{i=1}^{p} j_{i}^{*}\right)+\sum_{i=1}^{k} j_{i}^{*}} x, T^{n\left(\sum_{i=1}^{p} j_{i}^{*}\right)+\sum_{i=1}^{k} j_{i}^{*}-1} x\right) \leq D_{k} \\
\exists \lim _{n \rightarrow \infty} d\left(T^{n\left(\sum_{i=1}^{p} j_{i}^{*}\right)+\sum_{i=1}^{k} j_{i}^{*}+1} x, T^{n\left(\sum_{i=1}^{p} j_{i}^{*}\right)+\sum_{i=1}^{k} j_{i}^{*}} x\right) \\
\forall k \\
\forall k \in \bar{p} ;
\end{array}
$$

$$
\begin{aligned}
& \limsup _{n \rightarrow \infty} d\left(T^{n\left(\sum_{i=1}^{p} j_{i}^{*}\right)+\sum_{i=1}^{k+1} j_{i}^{*}+j+1} x, T^{n\left(\sum_{i=1}^{p} j_{i}^{*}\right)+\sum_{i=1}^{k+1} j_{i}^{*}+j} x\right) \\
& \quad=K_{k}^{1+j-j_{k}^{*}} D_{k} ; \quad j=1, \ldots, j_{k+1}^{*}-1, \quad \forall k \in \bar{p} ; \forall x \in \bigcup_{i \in \bar{p}} A_{i} .
\end{aligned}
$$

Proof. Take any $x \in A_{1}$. Thus, one gets from (9)

$d\left(T^{2} x, T x\right) \leq K_{1} d(x, T x)$

$$
\begin{aligned}
& d\left(T^{j_{1}^{*}} x, T^{j_{1}^{*}-1} x\right) \leq K_{1}^{j_{1}^{*}-1} d(x, T x) \\
& D_{1} \leq d\left(T^{j_{1}^{*}+1} x, T^{j_{1}^{*}} x\right) \leq K_{1}^{j_{1}^{*}} d(x, T x)+\left(1-K_{1}\right) D_{1} \\
& d\left(T^{j_{1}^{*}+2} x, T^{j_{1}^{*}+1} x\right) \leq K_{2} d\left(T^{j_{1}^{*}+1} x, T^{j_{1}^{*}} x\right) \\
& \quad \leq K_{2}\left[K_{1}^{j_{1}^{*}} d(x, T x)+\left(1-K_{1}\right) D_{1}\right] \\
& d\left(T^{j_{1}^{*}+j_{2}^{*}} x, T^{j_{1}^{*}+j_{2}^{*}-1} x\right) \leq K_{2}^{j_{2}^{*}-1}\left[K_{1}^{j_{1}^{*}} d(x, T x)+\left(1-K_{1}\right) D_{1}\right]
\end{aligned}
$$

$D_{2} \leq d\left(T^{j_{1}^{*}+j_{2}^{*}+1} x, T^{j_{1}^{*}+j_{2}^{*}} x\right)$

$$
\leq K_{2}^{j_{2}^{*}}\left[K_{1}^{j_{1}^{*}} d(x, T x)+\left(1-K_{1}\right) D_{1}\right]+\left(1-K_{2}\right) D_{2}
$$$$
d\left(T^{\sum_{i=1}^{p} j_{i}^{*}} x, T^{\sum_{i=1}^{p} j_{i}^{*}-1} x\right)
$$$$
\leq K_{p}^{j_{p}^{*}-1}\left(\left(\prod_{i=1}^{p-1}\left[K_{i}^{j_{i}^{*}}\right]\right) d(x, T x)\right.
$$

$$
\left.+\sum_{j=1}^{p-1}\left(\prod_{i=j+1}^{p-1}\left[K_{i}^{j_{i}^{*}}\right]\right)\left(1-K_{j}\right) D_{j}\right)
$$

$D_{p} \leq d\left(T^{\sum_{i=1}^{p} j_{i}^{*}+1} x, T^{\sum_{i=1}^{p} j_{i}^{*}} x\right) \leq K d(x, T x)+M$,

for all $x \in \bigcup_{i \in \bar{p}} A_{i}, j=0,1, \ldots, j_{k+1}^{*}-1$, for all $k \in \bar{p}$. 
where

$$
\begin{gathered}
K=\prod_{i=1}^{p}\left[K_{i}^{j_{i}^{*}}\right]<1 ; \\
M=\sum_{j=1}^{p}\left(\prod_{i=j+1}^{p}\left[K_{i}^{j_{i}^{*}}\right]\right)\left(1-K_{j}\right) D_{j} .
\end{gathered}
$$

Then, since $K<1$,

$$
\begin{aligned}
& D_{p} \leq d\left(T^{n\left(\sum_{i=1}^{p} j_{i}^{*}\right)+1} x, T^{n\left(\sum_{i=1}^{p} j_{i}^{*}\right)} x\right) \\
& \leq K^{n} d(x, T x)+\frac{1-K^{n}}{1-K} M, \\
& d\left(T^{n\left(\sum_{i=1}^{p} j_{i}^{*}\right)+j_{1}^{*}+1} x, T^{n\left(\sum_{i=1}^{p} j_{i}^{*}\right)+j_{1}^{*}} x\right) \\
& \quad \leq K_{1}^{j_{1}^{*}}\left(K^{n} d(x, T x)+\frac{1-K^{n}}{1-K} M\right)+\left(1-K_{1}\right) D_{1}
\end{aligned}
$$

with $T^{n\left(\sum_{i=1}^{p} j_{i}^{*}\right)+j_{1}^{*}+1} x \in A_{2}$. Now, since

$$
D_{0}=\frac{\sum_{j=1}^{p}\left(\prod_{i=j+1}^{p}\left[K_{i}^{j_{i}^{*}}\right]\right)\left(1-K_{j}\right) D_{j}}{1-\prod_{i=1}^{p}\left[K_{i}^{j_{i}^{*}}\right]}=\frac{M}{1-K},
$$

it follows that

$$
\begin{aligned}
& D_{p} \leq \limsup _{n \rightarrow \infty} d\left(T^{n\left(\sum_{i=1}^{p} j_{i}^{*}\right)+1} x, T^{n\left(\sum_{i=1}^{p} j_{i}^{*}\right)} x\right) \leq D_{0} \\
& \limsup _{n \rightarrow \infty} d\left(T^{n\left(\sum_{i=1}^{p} j_{i}^{*}\right)+2} x, T^{n\left(\sum_{i=1}^{p} j_{i}^{*}\right)+1} x\right) \leq K_{1} D_{0} \\
& \limsup _{n \rightarrow \infty} d\left(T^{n\left(\sum_{i=1}^{p} j_{i}^{*}\right)+j_{1}^{*}-1} x, T^{n\left(\sum_{i=1}^{p} j_{i}^{*}\right)+j_{1}^{*}-2} x\right) \leq K_{1}^{j_{1}^{*}-2} D_{0} \\
& \limsup _{n \rightarrow \infty} d\left(T^{n\left(\sum_{i=1}^{p} j_{i}^{*}\right)+j_{1}^{*}} x, T^{n\left(\sum_{i=1}^{p} j_{i}^{*}\right)+j_{1}^{*}-1} x\right) \leq K_{1}^{j_{1}^{*}-1} D_{0} \\
& D_{1} \leq \limsup _{n \rightarrow \infty} d\left(T^{n\left(\sum_{i=1}^{p} j_{i}^{*}\right)+j_{1}^{*}+1} x, T^{n\left(\sum_{i=1}^{p} j_{i}^{*}\right)+j_{1}^{*}} x\right) \\
& \quad \leq K_{1}^{j_{1}^{*}} D_{0}+\left(1-K_{1}\right) D_{1} .
\end{aligned}
$$

Thus, if $D_{1}=K_{1}^{j_{1}^{*}-1} D_{0}$, then one gets from the previous relationships

$$
\begin{aligned}
& \exists \lim _{n \rightarrow \infty} d\left(T^{n\left(\sum_{i=1}^{p} j_{i}^{*}\right)+j_{1}^{*}+1} x, T^{n\left(\sum_{i=1}^{p} j_{i}^{*}\right)+j_{1}^{*}} x\right)=D_{1} \\
& \limsup _{n \rightarrow \infty} d\left(T^{n\left(\sum_{i=1}^{p} j_{i}^{*}\right)+j_{1}^{*}} x, T^{n\left(\sum_{i=1}^{p} j_{i}^{*}\right)+j_{1}^{*}-1} x\right) \leq D_{1} \\
& \exists \lim _{n \rightarrow \infty} d\left(T^{n\left(\sum_{i=1}^{p} j_{i}^{*}\right)+j_{1}^{*}+1} x, T^{n\left(\sum_{i=1}^{p} j_{i}^{*}\right)+j_{1}^{*}} x\right) \\
& \leq K_{1}^{j_{1}^{*}} D_{0}+\left(1-K_{1}\right) D_{1}=D_{1} \\
& \limsup _{n \rightarrow \infty} d\left(T^{n\left(\sum_{i=1}^{p} j_{i}^{*}\right)+1+j_{1}^{*}+j} x, T^{n\left(\sum_{i=1}^{p} j_{i}^{*}\right)+j_{1}^{*}+j} x\right) \\
& \leq K_{1}^{j+j_{1}^{*}} D_{0}=K_{1}^{1+j} D_{1} ; \quad j=1, \ldots, j_{2}^{*}-1 .
\end{aligned}
$$

In the same way, if the constraint $D_{1}=K_{1}^{j_{1}^{*}-1} D_{0}$ is extended to $D_{1}=K_{k}^{j_{1}^{*}-1} D_{0} ; D_{k}=K_{k}^{j_{k}^{*}-1}\left(\prod_{i=1}^{k-1}\left[K_{i}^{j_{i}^{*}}\right]\right) D_{0}$; for all $k(\geq 2) \in \bar{p}$, then one gets from (34) and (38) that

$$
\begin{aligned}
& d\left(T^{n\left(\sum_{i=1}^{p} j_{i}^{*}\right)+\sum_{i=1}^{k} j_{i}^{*}+1} x, T^{n\left(\sum_{i=1}^{p} j_{i}^{*}\right)+\sum_{i=1}^{k} j_{i}^{*}} x\right) \\
& \quad \leq \prod_{i=1}^{k}\left[K_{i}^{j_{i}^{*}}\right]\left(K^{n} d(x, T x)+\frac{1-K^{n}}{1-K} M\right)+\left(1-K_{k}\right) D_{k} ;
\end{aligned}
$$

for all $k \in \bar{p}$. Thus,

$$
\begin{aligned}
D_{k} \leq & \limsup _{n \rightarrow \infty} d\left(T^{n\left(\sum_{i=1}^{p} j_{i}^{*}\right)+\sum_{i=1}^{k} j_{i}^{*}+1} x, T^{n\left(\sum_{i=1}^{p} j_{i}^{*}\right)+\sum_{i=1}^{k} j_{i}^{*}} x\right) \\
\leq & \lim _{n \rightarrow \infty}\left(\prod_{i=1}^{k}\left[K_{i}^{j_{i}^{*}}\right]\left(K^{n} d(x, T x)+\frac{1-K^{n}}{1-K} M\right)\right) \\
& +\left(1-K_{k}\right) D_{k} \\
= & \frac{\prod_{i=1}^{k}\left[K_{i}^{j_{i}^{*}}\right]}{1-K} M+\left(1-K_{k}\right) D_{k} \\
= & D_{0}\left(\prod_{i=1}^{k}\left[K_{i}^{j_{i}^{*}}\right]\right)+\left(1-K_{k}\right) D_{k} \\
= & K_{k} D_{k}+\left(1-K_{k}\right) D_{k}=D_{k} ; \quad \forall k \in \bar{p}
\end{aligned}
$$

with $T^{n\left(\sum_{i=1}^{p} j_{i}^{*}\right)+\sum_{i=1}^{k} j_{i}^{*}+1} x \in A_{k+1}$. Then, (31)-(33) follow and the result is proven for $x \in A_{1}$. Such a choice can be made with no loss in generality since, if, instead, $x \in A_{i}$ for any given $i \in \bar{p}$, then the previous result still holds with $T^{n\left(\sum_{i=1}^{p} j_{i}^{*}\right)+\sum_{i=1}^{k} j_{i}^{*}+1} x \in A_{k+1}$ with $k+1+i \equiv j(\bmod p)$ for a unique integer $j \in \bar{p}$. On the other hand (28)-(29) are a consequence of (36) which is independent of the constraints $D_{k}=K_{k}^{j_{k}^{*}-1}\left(\prod_{i=1}^{k-1}\left[K_{i}^{j_{i}^{*}}\right]\right) D_{0}$; for all $k \in \bar{p}$.

Remark 9. (1) Note that Theorem 8 requires a set of necessary constraints on the $K_{i}$ and $j_{i}^{*}$; for all $i \in \bar{p}$ which are induced by Assumptions 4-5 as follows:

$$
\begin{gathered}
\sigma_{i}=\frac{D_{i+1}}{D_{i}}=K_{i+1}^{j_{i+1}^{*}-1} K_{i} \quad(i=2,3, \ldots, p) \\
(1-K) D_{0} \\
=\left[\sum_{j=2}^{p}\left\{\left(\prod_{i=j+1}^{p}\left[K_{i}^{j_{i}^{*}}\right]\right)\left(1-K_{j}\right)\left(\prod_{k=1}^{j-1}\left[\sigma_{k}\right]\right)\right\}\right. \\
\left.+\prod_{i=2}^{p}\left[K_{i}^{j_{i}^{*}}\right]\left(1-K_{1}\right)\right] D_{1}
\end{gathered}
$$




$$
\begin{gathered}
=\left[\sum _ { j = 2 } ^ { p } \left\{\left(\prod_{i=j+1}^{p}\left[K_{i}^{j_{i}^{*}}\right]\right)\left(1-K_{j}\right)\right.\right. \\
\left.\times\left(\prod_{k=1}^{j-1}\left[K_{k+1}^{j_{k+1}^{*}-1} K_{k}\right]\right)\right\} \\
\left.+\prod_{i=2}^{p}\left[K_{i}^{j_{i}^{*}}\right]\left(1-K_{1}\right)\right] D_{1}
\end{gathered}
$$

with $K=\prod_{i=1}^{p}\left[K_{i}^{j_{i}^{*}}\right]$.

(2) Note that if $K_{i}=K<1, j_{i}^{*}=1$ and $D_{i}=D$; for all $i \in \bar{p}$, then $T: \bigcup_{i \in \bar{p}} A_{i} \rightarrow \bigcup_{i \in \bar{p}} A_{i}$ is a contractive $p$-cyclic and

$$
D_{0}=\frac{\sum_{i=0}^{p-1} K^{i}}{1-K^{p}}(1-K) D=\frac{1}{1-K^{p}} \frac{1-K^{p}}{1-K}(1-K) D=D .
$$

If (29) is evaluated in this case, then the limit superior is also the limit inferior of the obtained expression leading to the existence of the limit being equal to $D$, the distance inbetween al, the adjacent subsets $D_{i} \subset X$; for all $i \in \bar{p}$. This is a well- known result for contractive $p$-cyclic self-mappings $T: \bigcup_{i \in \bar{p}} A_{i} \rightarrow \bigcup_{i \in \bar{p}} A_{i},[23,24,42]$.

(3) Note that the first property of (32) is a convergence of the iterated sequences of distances to best proximity points of adjacent subsets provided that Assumptions 1-5 of Theorem 8 hold implying, in particular, a strict contractive constant on a composite self-mapping $T^{\sum_{i=1}^{p} j_{i}^{*}}: \bigcup_{i \in \bar{p}} A_{i} \rightarrow \bigcup_{i \in \bar{p}} A_{i}$ (see Assumption 3) and a set of further constraints on the distances in-between adjacent subsets even if such distances are not identical (see Assumptions 4-5). The following result is supported by parallel results in [42] (see also [43]) for cyclic self-mappings. It establishes the asymptotic convergence of the iterated sequences to cycles containing best proximity points in-between adjacent subsets in uniformly convex Banach spaces.

Theorem 10. Assume that $(X,\|\|)$ is a uniformly convex Banach space with the subsets $A_{i} \subset X$ being all disjoint, bounded, closed, and convex for all $i \in \bar{p}$ and that $T$ : $\bigcup_{i \in \bar{p}} A_{i} \rightarrow \bigcup_{i \in \bar{p}} A_{i}$ is a p-precyclic self-mapping subject to Assumptions 1-5 of Theorem 8.

Then, the subsequences $\left\{T^{n\left(\sum_{i=1}^{p} j_{i}^{*}\right)+\sum_{i=1}^{k} j_{i}^{*}} x\right\}_{n \in \mathbf{Z}_{0+}} \subseteq A_{k}$ and $\left\{T^{n\left(\sum_{i=1}^{p} j_{i}^{*}\right)+\sum_{i=1}^{k} j_{i}^{*}+1} x\right\}_{n \in \mathbf{Z}_{0+}} \subseteq A_{k+1}$ converge to respective unique best proximity points $x_{k}^{*}$ in $A_{k}$ and $x_{k+1}^{*}=T x_{k}^{*}$ in $A_{k+1}$ for each starting point $x \in A_{i}$; for all $i, k \in \bar{p}$. The sequence
$\left\{T^{n} x\right\}_{n \in \mathbf{Z}_{0+}}$ converges asymptotically to a unique cycle of $q=$ $\sum_{i=1}^{p} x_{i}^{*}$ points:

$$
\begin{aligned}
\widehat{x}^{*}:=\left(\bar{x}_{11}, \bar{x}_{12}=T \bar{x}_{11}, \ldots, \bar{x}_{1 j_{1}^{*}}=T^{j_{1}^{*}-1} \bar{x}_{11}=x_{1}^{*},\right. \\
\left.\bar{x}_{21}=T x_{1}^{*}, \ldots, x_{p-1}^{*}, \bar{x}_{p 1}=T x_{p-1}^{*}, \ldots, \bar{x}_{p j_{p}^{*}}=x_{p}^{*}\right)
\end{aligned}
$$

which contains the p best proximity points.

Proof (Outline of Proof). Note that $(X, d)$ is a complete metric space for a || || (norm-) induced metric $d: X \times X \rightarrow \mathbf{R}_{0+}$ since $(X,\|\|)$ is a Banach space. Thus, Theorem 8 remains true for such a metric. Since the subsets $A_{i} \subset X$ are nonempty, bounded, and closed (then compact and also boundedly compact), there exist $x_{i}^{*} \in A_{i}, T x_{i}^{*} A_{i+1}$ such that $d\left(x_{i}^{*}, T x_{i}^{*}\right)=\left\|x_{i}^{*}-T x_{i}^{*}\right\|=D_{i}$ for each $i \in \bar{p}$ so that $x_{i}^{*}$ and $T x_{i}^{*}$ are the best proximity points in $A_{i}$ to $A_{i+1}$ and $A_{i+1}$ from to $A_{i}$, respectively [42]. If $D_{i}=0$ and $j_{i}^{*}=1$, then both of them are confluent in the fixed point $x_{i}^{*}=T x_{i}^{*} \in A_{i} \cap A_{i+1}$; $i \in \bar{p}$. From the relation (32) of Theorem 8, it follows that

$$
\begin{array}{r}
\exists \lim _{n \rightarrow \infty} d\left(T^{n\left(\sum_{i=1}^{p} j_{i}^{*}\right)+\sum_{i=1}^{k} j_{i}^{*}+1} x, T^{n\left(\sum_{i=1}^{p} j_{i}^{*}\right)+\sum_{i=1}^{k} j_{i}^{*}} x\right)=D_{k} ; \\
\forall x \in A_{i} ; \forall i, k \in \bar{p} .
\end{array}
$$

The composite self-mapping $T^{\sum_{i=1}^{p} j_{i}^{*}}: \bigcup_{i \in \bar{p}} A_{i} \rightarrow$ $\bigcup_{i \in \bar{p}} A_{i}$ Lipschitz with constant $K=\prod_{i \in \bar{p}}\left[K_{i}^{\bar{j}_{i}^{*}}\right]$ everywhere in its definition domain $\bigcup_{i \in \bar{p}} A_{i}$. Thus, the limit of the distance and the distance of limits of the sequences $\left\{T^{n\left(\sum_{i=1}^{p} j_{i}^{*}\right)+\sum_{i=1}^{k} j_{i}^{*}} x\right\}_{n \in \mathbf{Z}_{0+}} \subseteq A_{k}$ and $\left\{T^{n\left(\sum_{i=1}^{p} j_{i}^{*}\right)+\sum_{i=1}^{k} j_{i}^{*}+1} x\right\}_{n \in \mathbf{Z}_{0+}} \subseteq A_{k+1}$ can be interchanged in (45) if the limits of such sequences exist. But such limits exist for any initial iteration point $x \in A_{i}$ since both sequences are Cauchy sequences and convergent since $(X, d) \equiv(X,\|\|)$ is a uniformly convex Banach space (Lemmas 3.7, 3.8 of [42]) The whole sequences and their limits are within the corresponding subsets since such subsets are closed and $(X, d) \equiv(X,\|\|)$ is complete. The uniqueness of the best proximity points in each of the subsets follows from the fact that the subsets $A_{i} \subset X$; for all $i \in \bar{p}$ are convex. The convergence of the sequences $\left\{T^{n} x\right\}_{n \in \mathbf{Z}_{0+}}$ to a unique cycle $\hat{x}^{*}$ of the form (45), containing $q=\sum_{i=1}^{p} x_{i}^{*}$ points, follows from the convergence of the above subsequences to unique best proximity points taking part of such a cycle and the fact that $T: \bigcup_{i \in \bar{p}} A_{i} \rightarrow \bigcup_{i \in \bar{p}} A_{i}$ is a single-valued self-mapping.

Example 11. Consider the following nonlinear difference sequence:

$$
\begin{array}{r}
x_{2 n+i-1}=\left(1-\alpha_{2 n+i-2}\right) x_{2 n+i-2}+\omega_{2 n+i-2}, \\
x_{2 n+j^{*}}=x_{2 m n+1}=-\beta_{2 m n} x_{2 m n} ; \quad \forall n \in \mathbf{Z}_{+} \\
\text {if } x_{1} \in A_{1}\left(\equiv \mathbf{R}_{0+}\right)
\end{array}
$$




$$
\begin{gathered}
x_{2 n+j^{*}+1}=x_{2 m n+2}=-\beta_{2 m n+1} x_{2 m n+1}, \\
x_{2 n+i}=\left(1-\alpha_{2 n+i-1}\right) x_{2 n+i-1}+\omega_{2 n+i-1} ; \quad \forall n \in \mathbf{Z}_{+} \\
\text {if } x_{1} \in \mathbf{R}_{0-}\left(\equiv-\mathbf{R}_{0+} \equiv A_{2}\right)
\end{gathered}
$$

for $i \in \overline{j^{*}}$ with $j^{*}=j_{1}^{*}=j_{n}^{*}=j_{n}^{*}(m)=2(m-1) n+1$, when $x_{n} \in A_{1} \equiv \mathbf{R}_{0-}$, and $j_{2}^{*}=1$ (that is, only one iteration remains in $A_{2} \equiv \mathbf{R}_{0-}$ before each switching to $\mathbf{R}_{0+}$ ) defined for some positive integer $m=m(n)$ under the following constraints:

$$
\begin{aligned}
& \left\{\omega_{n}\right\} \text { is a nonnegative real summable sequence } \\
& \left\{\alpha_{n}\right\} \subset[0,1] \text { is a real sequence, } \sum_{n=0}^{\infty} \alpha_{n}=\infty \text { and } \\
& \alpha_{n} \rightarrow 0 \text { as } n \rightarrow \infty \\
& \left\{\omega_{n}\right\} \subset \mathbf{R}_{0+} \text { is a real sequence and } \sum_{n=0}^{\infty} \omega_{n}<\infty \\
& \left\{\beta_{n}\right\} \subset[0, \bar{\beta}] \text { is a bounded real sequence fulfilling } \\
& \beta_{2 m n+2} \leq \beta_{2 n m+1} x_{2 n m+1} / x_{2 m n+2} \text {, for all } n \in \mathbf{Z}_{+} \text {if } \\
& x_{1} \in \mathbf{R}_{0+} \text { and } \beta_{2 m n+1} \leq \beta_{2 m n-1} x_{2 m n-1} / x_{2 m n+1} \text {; for all } \\
& n \in \mathbf{Z}_{+} \text {, otherwise. }
\end{aligned}
$$

Note that the difference equation is generated by a 2-precyclic self-mapping on $\mathbf{R}=\mathbf{R}_{0+} \cup \mathbf{R}_{0-}$ defined by the solution got from any initial condition. If $m=j_{n}^{*}=1$, then the solution point simply alternates in-between the subsets $\mathbf{R}_{0+}$ and $\mathbf{R}_{0_{-}}$ of $\mathbf{R}$ and the mapping becomes a 2-cyclic self-mapping. If $m>1$, the solution remains $j_{n}^{*}=2(m-1) n+1$ consecutive iterations in $\mathbf{R}_{0+}$ after entering it before the next switching to $\mathbf{R}_{\text {_ }}$ if $m(n)$ is infinity; for all $n \geq n_{0}$ and some finite $n_{0} \in \mathbf{Z}_{0+}$, then the solution remains in $\mathbf{R}_{0+}$ after a given finite iteration. The following nonexpansive condition holds:

$$
\begin{aligned}
\left|x_{2 n+j^{*}(n)+1}\right| \leq & \beta_{2 m(n) n+1}\left(\prod_{i=2 n-1}^{2 m(n)}\left[1-\alpha_{i}\right]\right)\left|x_{2 n}\right| \\
& +\sum_{i=2 n-1}^{2 m(n)} \prod_{j=i+1}^{2 m(n)}\left[1-\alpha_{j}\right] \omega_{2 i}
\end{aligned}
$$

for all $n \geq n_{1}$ since $\left\{\omega_{n}\right\}$ is nonnegative, summable, and then converges to zero, so that it has some strictly decreasing subsequence $\left\{\omega_{n}\right\}$ and then $\omega_{2 n}-\omega_{2 n-1}<0$; for all $n \geq n_{1}$ for some finite positive integer $n_{1}$. It follows, since $\alpha_{n} \rightarrow 0$ as $n \rightarrow \infty$, that

(a) if $\beta_{n} \rightarrow 1$ as $n \rightarrow \infty$, then the solution is weakly 2-precyclic asymptotically nonexpansive since $\alpha_{n} \rightarrow 0$ and $\omega_{n} \rightarrow 0$ as $n \rightarrow \infty$ and then $\lim \sup _{n \rightarrow \infty}\left(\left|x_{2 n+j^{*}(n)+1}\right|-\right.$ $\left.\left|x_{2 n}\right|\right) \leq 0, \lim \inf _{n \rightarrow \infty}\left(\left|x_{2 n+j^{*}(n)+1}\right|-\left|x_{2 n}\right|\right) \geq 0$ and $\left\{x_{n}\right\}$ converges.

If $j_{n}^{*}(x)=1$ for any $n \in \mathbf{Z}_{0+}$ and $x \in \mathbf{R}$, then the mapping defining the solution is also 2 -cyclic asymptotically nonexpansive.

(b) If $\beta_{n} \rightarrow 1$ as $n \rightarrow \infty$ and there are no subsequences of $\left\{\omega_{n}\right\}$ and $\left\{\alpha_{n}\right\}$ being simultaneously zero, then the solution is 2-precyclic (2-cyclic if $j_{n}^{*}$ is identically unity) weakly asymptotically contractive so that it converges to zero which is the fixed point being a confluent best proximity point of $\mathbf{R}_{0+}$ and $\mathbf{R}_{0-}$.
If $\alpha_{n} \rightarrow \alpha \in(0,1)$ or if $\lim _{\sup _{n \rightarrow \infty}} \alpha_{n} \leq \bar{\alpha}<1$, then the solution is 2-precyclic (2-cyclic if $j_{n}^{*}$ is identically unity) strongly asymptotically contractive so that it converges to zero. If there is some finite positive integer $n_{0}$ such that $j_{n_{0}}^{*}$ is infinite, then the solution is permanent and nonnegative in $\mathbf{R}_{0+}$ after a finite number of iterations and converges asymptotically to zero.

It is now proven that, in the most general considered case when the solution mapping is weakly 2-precyclic asymptotically nonexpansive, the fixed point (which is also stable equilibrium point and best proximity point on both subsets) is $x=0$.

Define $\bar{x}_{1}=x_{1}$, if $x_{1} \in \mathbf{R}_{0+}$ and $\bar{x}_{1}=x_{2}$, then $\bar{x}_{0} \in \mathbf{R}_{0+}$, if $x_{1} \in \mathbf{R}_{-}$and build the sequence $\left\{\bar{x}_{n}\right\}$ by $\bar{x}_{n}=x_{n}$ if $x_{n} \geq 0$ and $\bar{x}_{n}=0$, otherwise. Then, one gets

$$
-\infty<-\bar{x}_{1}=\lim _{n \rightarrow \infty}\left(\bar{x}_{n+1}-\bar{x}_{1}\right)=\lim _{n \rightarrow \infty}\left(\sum_{i=1}^{n}\left(\omega_{i}-\alpha_{i} \bar{x}_{i}\right)\right)
$$

so that $\sum_{n=1}^{\infty}\left(\alpha_{n} \bar{x}_{n}-\omega_{n}\right)=\bar{x}_{1}<\infty$. Since $\left\{\omega_{n}\right\}$ is summable and $\sum_{i=1}^{\infty} \alpha_{n}=\infty$, one gets

$$
\begin{aligned}
\left(\min _{n \in \mathbf{Z}_{+}} \bar{x}_{n}\right)\left(\sum_{i=1}^{\infty} \alpha_{n}\right) & \leq \sum_{i=1}^{\infty} \alpha_{n} \bar{x}_{n}=\bar{x}_{1}+\sum_{n=1}^{\infty} \omega_{n}<\infty \\
& \Longrightarrow \min _{n \in \mathbf{Z}_{+}} \bar{x}_{n}=\min _{n \in \mathbf{Z}_{+}} x_{n}=0 .
\end{aligned}
$$

Also, $\max _{n \in \mathbf{Z}_{-}}\left(\left|x_{n}\right|\right) \leq \lim _{n \rightarrow \infty}\left(\beta_{n}\right) \min _{n \in \mathbf{Z}_{0+}} x_{n} \leq$ $\min _{n \in \mathbf{Z}_{0+}} x_{n}=0$. As a result, the fixed point, and also equilibrium point, is $x=0$ and $x_{n} \rightarrow 0$ as $n \rightarrow \infty$ for any initial condition $x_{1} \in \mathbf{R}$.

\section{Acknowledgments}

The author is very grateful to the Spanish Government for its support of this research through Grant DPI2012-30651 and to the Basque Government for its support of this research through Grants IT378-10 and SAIOTEK S-PE12UN015. He is also grateful to the University of Basque Country for its financial support through Grant UFI 2011/07. He is also grateful to the reviewers for their interesting comments.

\section{References}

[1] Y. Yao, M. A. Noor, Y.-C. Liou, and S. M. Kang, "Iterative algorithms for general multivalued variational inequalities," Abstract and Applied Analysis, vol. 2012, Article ID 768272, 10 pages, 2012.

[2] M. De la Sen, "Stable iteration procedures in metric spaces which generalize a Picard-type iteration," Fixed Point Theory and Applications, vol. 2010, Article ID 953091, 15 pages, 2010.

[3] M. Ratchagit and K. Ratchagit, "Asymptotic stability and stabilization of fixed points for iterative sequence," International Journal of Research and Reviews in Computer Science, vol. 2, no. 4, pp. 987-989, 2011.

[4] D. Doric and R. Lazović, "Some Suzuki-type fixed point theorems for generalized multivalued mappings and applications," Fixed Point Theory and Applications, vol. 2011, article 40, 2011. 
[5] L.J. Ciric, "Multi-valued nonlinear contraction mappings," Nonlinear Analysis. Theory, Methods \& Applications A, vol. 71, no. 7-8, pp. 2716-2723, 2009.

[6] L.J. Ciric, "Fixed points for generalized multi-valued contractions," Matematički Vesnik, vol. 9, no. 24, pp. 265-272, 1972.

[7] S. L. Singh, S. N. Mishra, and S. Jain, "Round-off stability for multi-valued maps," Fixed Point Theory and Applications, vol. 2012, article 12, 2012.

[8] S. L. Singh, S. N. Mishra, R. Chugh, and R. Kamal, "General common fixed point theorems and applications," Journal of Applied Mathematics, vol. 2012, Article ID 902312, 14 pages, 2012.

[9] W. Laowang and B. Panyanak, "Common fixed points for some generalized multivalued nonexpansive mappings in uniformly convex metric spaces," Fixed Point Theory and Applications, vol. 2011, article 20, 2011.

[10] H. Khandani, S. M. Vaezpour, and B. Sims, "Common fixed points of generalized multivalued contraction on complete metric spaces," Journal of Computational Analysis and Applications, vol. 13, no. 6, pp. 1025-1038, 2011.

[11] M. Abbas, "Coincidence points of multivalued $f$-almost nonexpansive mappings," Fixed Point Theory, vol. 13, no. 1, pp. 3-10, 2012.

[12] Sh. Rezapour and P. Amiri, "Fixed point of multivalued operators on ordered generalized metric spaces," Fixed Point Theory, vol. 13, no. 1, pp. 173-178, 2012.

[13] A. Petruşel and G. Petruşel, "Multivalued Picard operators," Journal of Nonlinear and Convex Analysis, vol. 13, no. 1, pp. 157171, 2012.

[14] T. P. Petru, A. Petruşel, and J.-C. Yao, "Ulam-Hyers stability for operatorial equations and inclusions via nonself operators," Taiwanese Journal of Mathematics, vol. 15, no. 5, pp. 2195-2212, 2011.

[15] H. K. Nashine and W. Shatanawi, "Coupled common fixed point theorems for a pair of commuting mappings in partially ordered complete metric spaces," Computers \& Mathematics with Applications, vol. 62, no. 4, pp. 1984-1993, 2011.

[16] K. Iséki, “On common fixed points of mappings," Bulletin of the Australian Mathematical Society, vol. 10, pp. 365-370, 1974.

[17] K. Iseki, "Multi-valued contraction mappings in complete metric spaces," Rendiconti del Seminario Matematico della Universita di Padova, vol. 53, pp. 15-19, 1975.

[18] A. Rashid Butt, Fixed points of set valued maps [Ph.D. thesis], Department of Mathematics, Lahore University of Management Sciences, Lahore, Pakistan, 2010.

[19] I. Beg and A. R. Butt, "Common fixed point for generalized set valued contractions satisfying an implicit relation in partially ordered metric spaces," Mathematical Communications, vol. 15, no. 1, pp. 65-76, 2010.

[20] M. Abbas, A. R. Khan, and T. Nazir, "Coupled common fixed point results in two generalized metric spaces," Applied Mathematics and Computation, vol. 217, no. 13, pp. 6328-6336, 2011.

[21] M. Abbas, M. Ali Khan, and S. Radenović, "Common coupled fixed point theorems in cone metric spaces for $w$-compatible mappings," Applied Mathematics and Computation, vol. 217, no. 1, pp. 195-202, 2010.

[22] D. R. Sahu, S. M. Kang, and V. Sagar, "Approximation of common fixed points of a sequence of nearly nonexpansive mappings and solutions of variational inequality problems," Journal of Applied Mathematics, vol. 2012, Article ID 902437, 12 pages, 2012.
[23] S. Karpagam and S. Agrawal, "Best proximity point theorems for $p$-cyclic Meir-Keeler contractions," Fixed Point Theory and Applications, vol. 2009, Article ID 197308, 9 pages, 2009.

[24] M. De la Sen, "Linking contractive self-mappings and cyclic Meir-Keeler contractions with Kannan self-mappings," Fixed Point Theory and Applications, vol. 2010, Article ID 572057, 23 pages, 2010.

[25] M. Kikkawa and T. Suzuki, "Three fixed point theorems for generalized contractions with constants in complete metric spaces," Nonlinear Analysis. Theory, Methods \& Applications A, vol. 69, no. 9, pp. 2942-2949, 2008.

[26] Y. Enjouji, M. Nakanishi, and T. Suzuki, "A generalization of Kannan's fixed point theorem," Fixed Point Theory and Applications, vol. 2009, Article ID 192872, 10 pages, 2009.

[27] M. S. Khan, "Common fixed point theorems for multivalued mappings," Pacific Journal of Mathematics, vol. 95, no. 2, pp. 337347, 1981.

[28] Y. J. Cho, J. K. Kim, and S. M. Kang, Fixed Point Theory and Applications, vol. 3, Nova Publishers, 2002.

[29] A. Ashyralyev and H. O. Fattorini, "On uniform difference schemes for second-order singular perturbation problems in Banach spaces," SIAM Journal on Mathematical Analysis, vol. 23, no. 1, pp. 29-54, 1992.

[30] B. T. Kalimbetov, M. A. Temirbekov, and Z. O. Khabibullayev, "Asymptotic solutions of singular perturbed problems with an instable spectrum of the limiting operator," Abstract and Applied Analysis, vol. 2012, Article ID 120192, 16 pages, 2012.

[31] V. P. Maksimov and A. L. Chadov, "A class of controls for a functional-differential continuous-discrete system," Russian Mathematics, vol. 56, no. 9, pp. 62-65, 2012.

[32] A. Ashyralyev and Y. A. Sharifov, "Existence and uniqueness of solutions for the system of nonlinear fractional differential equations with nonlocal and integral boundary conditions," Abstract and Applied Analysis, vol. 2012, Article ID 594802, 14 pages, 2012.

[33] M. De la Sen, "About robust stability of Caputo linear fractional dynamic systems with time delays through fixed point theory," Fixed Point Theory and Applications, vol. 2011, Article ID 867932, 19 pages, 2011.

[34] D. Amanov and A. Ashyralyev, "Initial-boundary value problem for fractional partial differential equations of higher order," Abstract and Applied Analysis, vol. 2012, Article ID 973102, 16 pages, 2012.

[35] M. de la Sen, "Application of the nonperiodic sampling to the identifiability and model matching problems in dynamic systems," International Journal of Systems Science, vol. 14, no. 4, pp. 367-383, 1983.

[36] M. de la Sen, "Stability of composite systems with an asymptotically hyperstable subsystem," International Journal of Control, vol. 44, no. 6, pp. 1769-1775, 1986.

[37] H. Xiang and J. Cao, "Periodic oscillation of fuzzy CohenGrossberg neural networks with distributed delay and variable coefficients," Journal of Applied Mathematics, vol. 2008, Article ID 453627, 18 pages, 2008.

[38] M. De la Sen, R. P. Agarwal, A. Ibeas, and S. Alonso-Quesada, "On a generalized time-varying SEIR epidemic model with mixed point and distributed time-varying delays and combined regular and impulsive vaccination controls," Advances in Difference Equations, vol. 2010, Article ID 281612, 42 pages, 2010.

[39] Y. Zhang, "Asymptotic stability of impulsive reaction-diffusion cellular neural networks with time-varying delays," Journal of Applied Mathematics, vol. 2010, Article ID 501891, 17 pages, 2012. 
[40] L.-C. Ceng and J.-C. Yao, "A hybrid iterative scheme for mixed equilibrium problems and fixed point problems," Journal of Computational and Applied Mathematics, vol. 214, no. 1, pp. 186201, 2008.

[41] N. Hussain and M. A. Khamsi, "On asymptotic pointwise contractions in metric spaces," Nonlinear Analysis. Theory, Methods \& Applications A, vol. 71, no. 10, pp. 4423-4429, 2009.

[42] A. A. Eldred and P. Veeramani, "Existence and convergence of best proximity points," Journal of Mathematical Analysis and Applications, vol. 323, no. 2, pp. 1001-1006, 2006.

[43] I. A. Pyatyshev, "Operations on approximatively compact sets," Mathematical Notes, vol. 82, no. 5, pp. 729-735, 2007. 


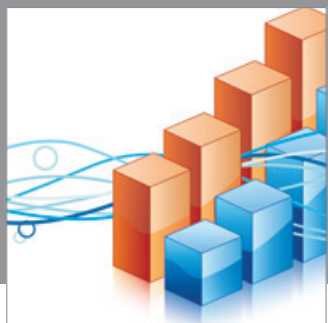

Advances in

Operations Research

mansans

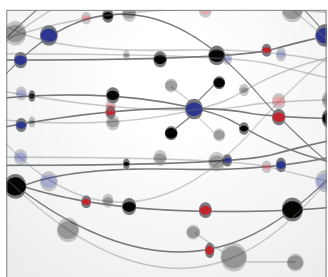

The Scientific World Journal
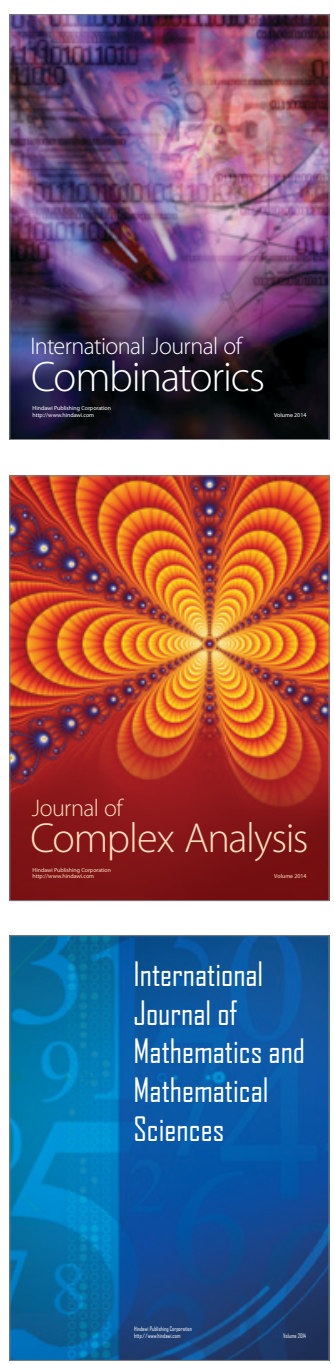
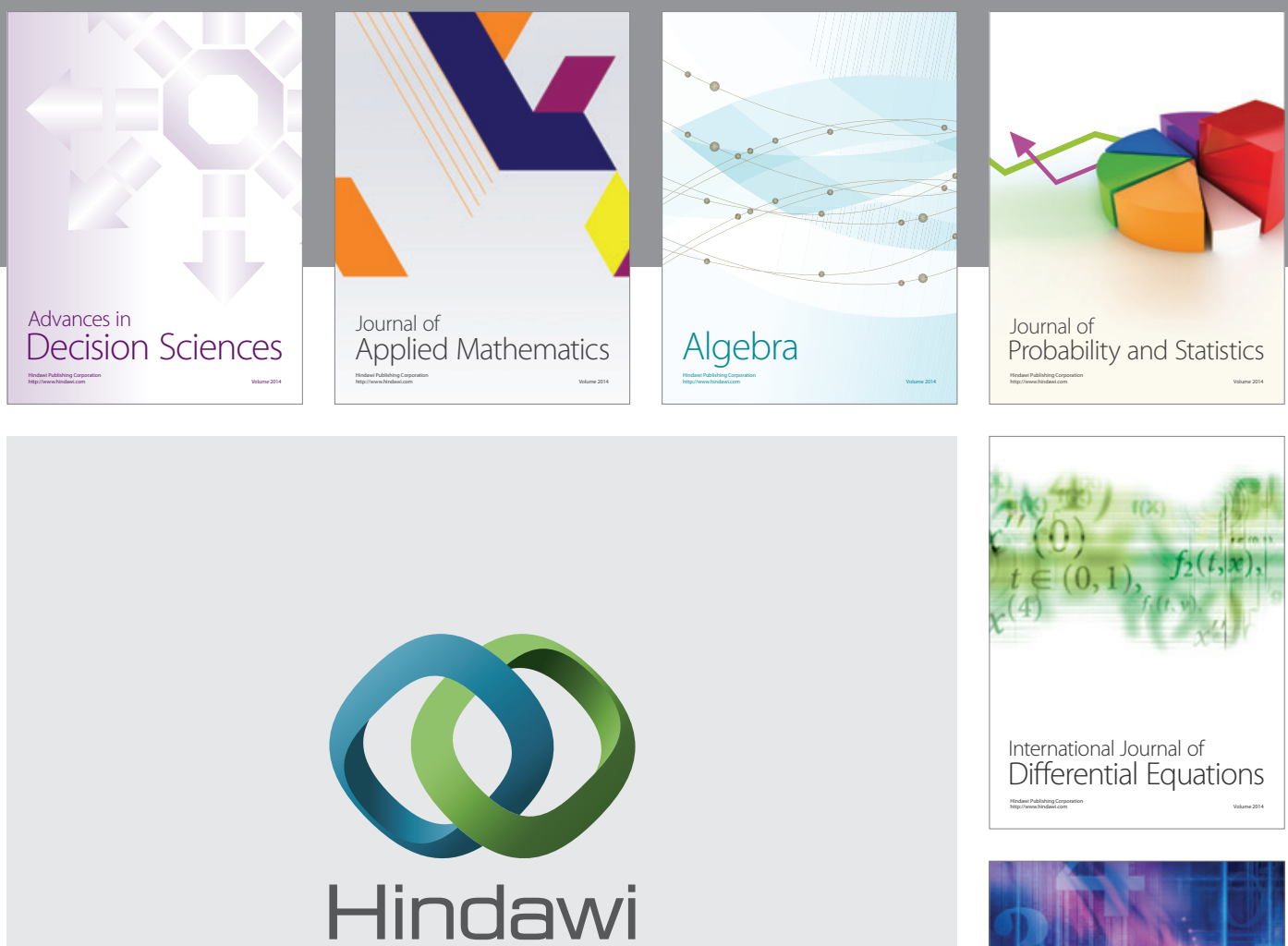

Submit your manuscripts at http://www.hindawi.com
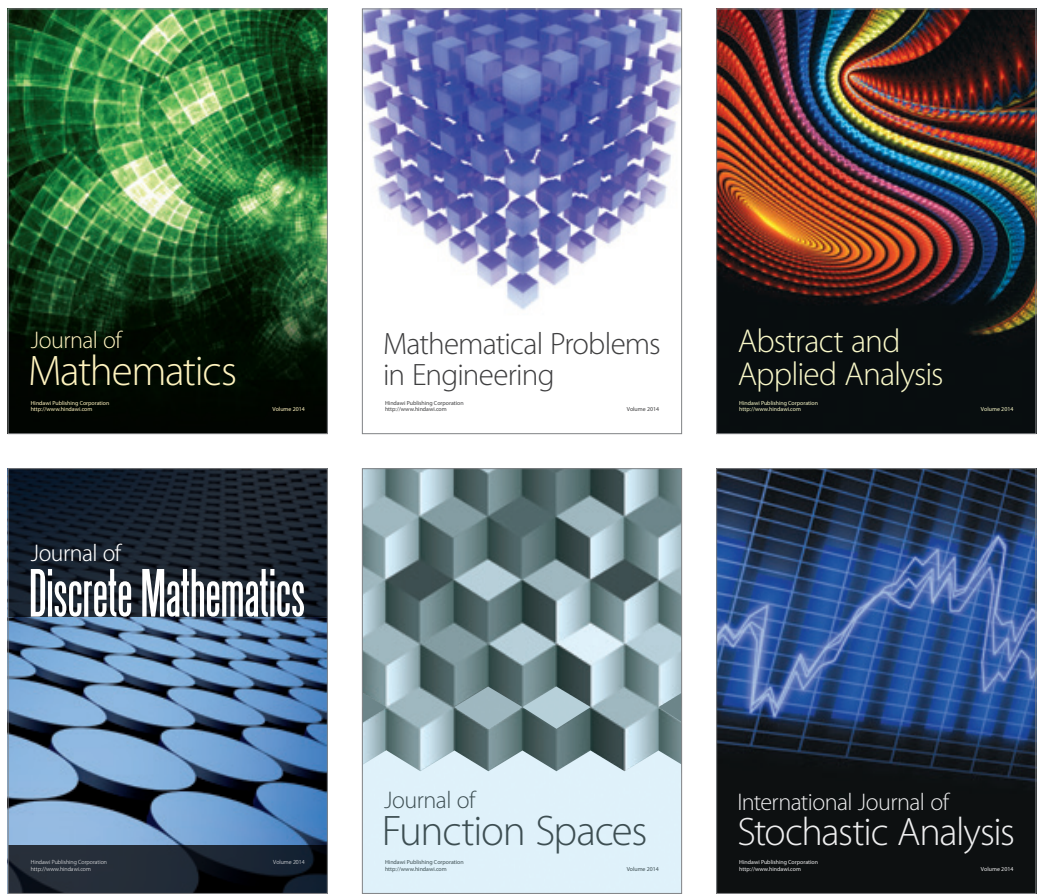

Journal of

Function Spaces

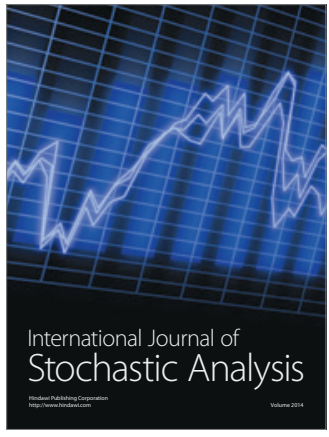

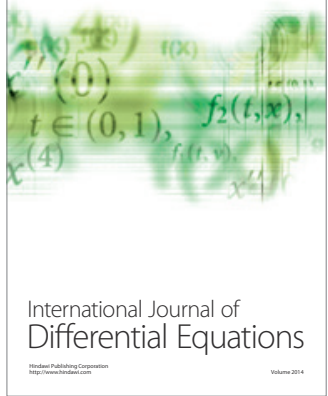
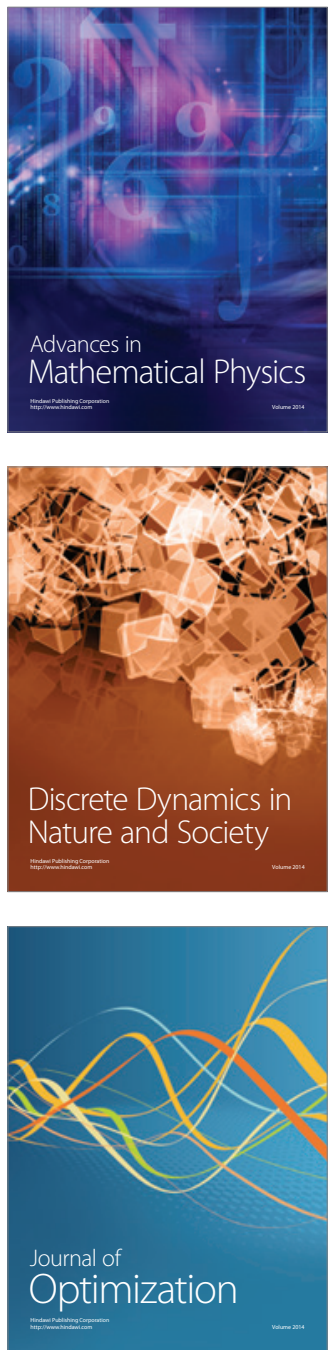